\title{
Fermion EDMs with minimal flavor violation
}

\author{
Xiao-Gang He, ${ }^{a, b, c}$ Chao-Jung Lee, ${ }^{c}$ Siao-Fong $\mathbf{L i}^{c}$ and Jusak Tandean ${ }^{c}$ \\ ${ }^{a} I N P A C$, SKLPPC, and Department of Physics, Shanghai Jiao Tong University, \\ Shanghai 200240, China \\ ${ }^{b}$ Physics Division, National Center for Theoretical Sciences, Department of Physics, \\ National Tsing Hua University, Hsinchu 300, Taiwan \\ ${ }^{c}$ CTS, CASTS, and Department of Physics, National Taiwan University, \\ Taipei 106, Taiwan \\ E-mail: hexg@phys.ntu.edu.tw, r02222074@ntu.edu.tw, \\ r01222004@ntu.edu.tw, jtandean@yahoo.com
}

Abstract: We study the electric dipole moments (EDMs) of fermions in the standard model supplemented with right-handed neutrinos and its extension including the neutrino seesaw mechanism under the framework of minimal flavor violation (MFV). In the quark sector, we find that the current experimental bound on the neutron EDM does not yield a significant restriction on the scale of MFV. In addition, we consider how MFV may affect the contribution of the strong theta-term to the neutron EDM. For the leptons, the existing EDM data also do not lead to strict limits if neutrinos are Dirac particles. On the other hand, if neutrinos are Majorana in nature, we find that the constraints become substantially stronger. Moreover, the results of the latest search for the electron EDM by the ACME Collaboration are sensitive to the MFV scale of order a few hundred GeV or higher. We also look at constraints from $C P$-violating electron-nucleon interactions that have been probed in atomic and molecular EDM searches.

Keywords: Beyond Standard Model, Neutrino Physics, CP violation, Electromagnetic Processes and Properties

ARXiV EPRINT: 1404.4436 


\section{Contents}

1 Introduction 1

2 Minimal flavor violation framework $\quad 2$

3 Fermion EDMs in MFV framework $\quad 6$

4 Numerical analysis $\quad 8$

4.1 Neutron EDM 8

$\begin{array}{llr}4.2 & \text { MFV contribution to strong theta term } & 10\end{array}$

$\begin{array}{lll}4.3 & \text { Electron EDM } & 11\end{array}$

$\begin{array}{lll}4.4 & \text { Muon and tau EDMs } & 18\end{array}$

$4.5 C P$-violating electron-neutron interactions 20

4.6 Muon $g-2, \mu \rightarrow e \gamma$, nuclear $\mu \rightarrow e$ conversion, $\bar{B} \rightarrow X_{s} \gamma \quad 21$

5 Conclusions $\quad 24$

A Evaluation of some products of A and B matrices 24

\section{Introduction}

Searches for electric dipole moments (EDMs) are a powerful means of probing new sources of the violation of charge parity $(C P)$ and time reversal $(T)$ symmetries beyond the standard model (SM) of particle physics [1-6]. Recently the ACME experiment [7], which utilized the polar molecule thorium monoxide to look for the EDM of the electron, $d_{e}$, has produced a new result of $d_{e}=\left(-2.1 \pm 3.7_{\text {stat }} \pm 2.5_{\text {syst }}\right) \times 10^{-29} e \mathrm{~cm}$, which corresponds to an upper limit of $\left|d_{e}\right|<8.7 \times 10^{-29} \mathrm{ecm}$ at $90 \%$ confidence level (CL). This is more stringent than the previous best bound by about an order of magnitude, but still way above the SM expectation for $d_{e}$, which is at the level of $10^{-44} e \mathrm{~cm}$ [8]. Hence there is abundant room between the current limit and SM value of $d_{e}$ where potential new physics may be observed in future measurements. In the quark sector, the EDM of the neutron, $d_{n}$, plays an analogous role in the quest of new physics. At present its experimental limit is $\left|d_{n}\right|<2.9 \times 10^{-26} e \mathrm{~cm}$ at $90 \%$ CL [9], while the SM predicts it to be in the range of $10^{-32}-10^{-31}$ e cm [10-13].

Extra ingredients beyond the SM can increase the electron and neutron EDMs tremendously with respect to their SM predictions, even up to their existing measured bounds. Such substantial enlargement may have various causes which could greatly differ from model to model. It is, therefore, of interest to analyze fermion EDMs arising from possible nonstandard origins under a framework that allows one to deal with some general features 
of the physics without getting into model specifics. This turns out to be feasible under the context of the so-called minimal flavor violation (MFV) which presupposes that the sources of all flavor-changing neutral currents (FCNC) and $C P$ violation reside in renormalizable Yukawa couplings defined at tree level [14-22]. Thus the MFV framework offers a systematic way to explore SM-related new interactions which do not conserve flavor and $C P$ symmetries.

In an earlier paper [23], motivated by the recent ACME data, we have adopted the MFV hypothesis in order to examine $d_{e}$ in the SM slightly expanded with the inclusion of three right-handed neutrinos and in its extension incorporating the seesaw mechanism for light neutrino mass generation. In the present work, we would like to provide a more extensive treatment of our previous study, covering the EDMs of the other charged leptons as well. For $d_{e}$ particularly, we demonstrate in greater detail how various factors may affect it within the MFV context, taking into account extra empirical information on neutrino masses. Moreover, we address the possibility that $d_{e}$ is correlated with the effective Majorana mass that is testable in ongoing and upcoming searches for neutrinoless double-beta decay. We will also perform an MFV analysis on the quark EDMs and estimate the resulting neutron EDM. In addition, we consider the MFV effect on the contribution of the theta term in QCD to the neutron EDM.

The structure of the paper is as follows. In section 2, we describe the MFV framework and its aspects which are relevant to our evaluation of fermion EDMs as probes for the scale of MFV. In section 3 we derive the expressions for quark and lepton EDMs from several effective operators satisfying the MFV principle. Section 4 contains our numerical analysis. After determining the neutron EDM from the quark contributions and inferring the constraint on the MFV scale from the neutron data, we examine how the contribution of the QCD theta-term is altered in the presence of MFV. In the lepton sector, we devote much of our attention to the electron EDM in light of the ACME data and briefly address its muon and tau counterparts. The acquired constraints on the MFV scale depend considerably on whether the light neutrinos are Dirac or Majorana in nature, the EDMs in the former case being much smaller than the latter. Subsequently, we look at $C P$-violating electron-nucleon interactions, which were also investigated by ACME and other experiments looking for atomic or molecular EDMs. Lastly, we discuss potential constraints from flavor-changing and other flavor-conserving processes. We make our conclusions in section 5. An appendix collects some useful lengthy formulas.

\section{Minimal flavor violation framework}

In the SM supplemented with three right-handed neutrinos, the renormalizable Lagrangian for the quark and lepton masses can be expressed as

$$
\begin{aligned}
\mathcal{L}_{\mathrm{m}}= & -\bar{Q}_{k, L}\left(Y_{u}\right)_{k l} U_{l, R} \tilde{H}-\bar{Q}_{k, L}\left(Y_{d}\right)_{k l} D_{l, R} H-\bar{L}_{k, L}\left(Y_{\nu}\right)_{k l} \nu_{l, R} \tilde{H}-\bar{L}_{k, L}\left(Y_{e}\right)_{k l} E_{l, R} H \\
& -\frac{1}{2} \bar{\nu}_{k, R}^{c}\left(M_{\nu}\right)_{k l} \nu_{l, R}+\text { H.c. },
\end{aligned}
$$

where summation over $k, l=1,2,3$ is implicit, $Q_{k, L}\left(L_{k, L}\right)$ represents left-handed quark (lepton) doublets, $U_{k, R}$ and $D_{k, R}\left(\nu_{k, R}\right.$ and $\left.E_{k, R}\right)$ denote right-handed up- and down-type 
quarks (neutrinos and charged leptons), respectively, $Y_{u, d, \nu, e}$ are matrices containing the Yukawa couplings, $M_{\nu}$ is the Majorana mass matrix of the right-handed neutrinos, $H$ is the Higgs doublet, and $\tilde{H}=i \tau_{2} H^{*}$ involving the second Pauli matrix $\tau_{2}$. The Higgs' vacuum expectation value $v \simeq 246 \mathrm{GeV}$ breaks the electroweak symmetry as usual, which makes the weak gauge bosons and charged leptons massive and also induces Dirac mass terms for the neutrinos. The $M_{\nu}$ part in $\mathcal{L}_{\mathrm{m}}$ plays an essential role in the type-I seesaw mechanism [24-32]. ${ }^{1}$ If neutrinos are Dirac particles, however, the $M_{\nu}$ terms are absent.

For the quark sector, the MFV hypothesis [21] implies that the Lagrangian in eq. (2.1) is formally invariant under the global group $\mathrm{U}(3)_{Q} \times \mathrm{U}(3)_{U} \times \mathrm{U}(3)_{D}=G_{q} \times \mathrm{U}(1)_{Q} \times \mathrm{U}(1)_{U} \times$ $\mathrm{U}(1)_{D}$, where $G_{q}=\mathrm{SU}(3)_{Q} \times \mathrm{SU}(3)_{U} \times \mathrm{SU}(3)_{D}$. This entails that the three generations of $Q_{k, L}, U_{k, R}$, and $D_{k, R}$ transform as fundamental representations of the $\mathrm{SU}(3)_{Q, U, D}$, respectively, namely

$$
Q_{L} \rightarrow V_{Q} Q_{L}, \quad U_{R} \rightarrow V_{U} U_{R}, \quad D_{R} \rightarrow V_{D} D_{R}, \quad V_{Q, U, D} \in \mathrm{SU}(3)
$$

Moreover, the Yukawa couplings are taken to be spurions which transform according to

$$
Y_{u} \rightarrow V_{Q} Y_{u} V_{U}^{\dagger}, \quad Y_{d} \rightarrow V_{Q} Y_{d} V_{D}^{\dagger}
$$

Consequently, to arrange nontrivial FCNC and $C P$-violating interactions satisfying the MFV principle and involving no more than two quarks, one puts together an arbitrary number of the Yukawa coupling matrices $Y_{u} \sim(3, \overline{3}, 1)$ and $Y_{d} \sim(3,1, \overline{3})$ as well as their Hermitian conjugates to set up the $G_{q}$ representations $\Delta_{q} \sim(1 \oplus 8,1,1)$, $\Delta_{u 8} \sim(1,1 \oplus 8,1), \quad \Delta_{d 8} \sim(1,1,1 \oplus 8), \Delta_{u} \sim(\overline{3}, 3,1)$, and $\Delta_{d} \sim(\overline{3}, 1,3)$, combines them with two quark fields to build the $G_{q}$-invariant objects $\bar{Q}_{L} \gamma_{\alpha} \Delta_{q} Q_{L}, \bar{U}_{R} \gamma_{\alpha} \Delta_{u 8} U_{R}$, $\bar{D}_{R} \gamma_{\alpha} \Delta_{d 8} D_{R}, \bar{U}_{R}\left(1, \sigma_{\alpha \beta}\right) \Delta_{u} Q_{L}$, and $\bar{D}_{R}\left(1, \sigma_{\alpha \beta}\right) \Delta_{d} Q_{L}$, includes appropriate numbers of the Higgs and gauge fields to arrive at singlets under the SM gauge group, and contracts all the Lorentz indices. Since $\bar{Q}_{L} \gamma_{\alpha} \Delta_{q} Q_{L}, \bar{U}_{R} \gamma_{\alpha} \Delta_{u 8} U_{R}$, and $\bar{D}_{R} \gamma_{\alpha} \Delta_{d 8} D_{R}$ in this case must be Hermitian, $\Delta_{q, u 8, d 8}$ must be Hermitian as well.

The Lagrangian describing the EDM $d_{f}$ of a fermion $f$ is $\mathcal{L}_{f \text { edm }}=-\frac{i}{2} d_{f} \bar{f} \sigma^{\kappa \omega} \gamma_{5} f F_{\kappa \omega}$, where $F_{\kappa \omega}$ is the photon field strength tensor. Accordingly, among the combinations listed in the preceding paragraph, only $\bar{U}_{R} \sigma_{\alpha \beta} \Delta_{u} Q_{L}$ and $\bar{D}_{R} \sigma_{\alpha \beta} \Delta_{d} Q_{L}$ pertain to our examination of quark EDMs. For $\Delta_{u, d}$, one can take $\Delta_{u}=Y_{u}^{\dagger} \Delta$ and $\Delta_{d}=Y_{d}^{\dagger} \Delta$, where $\Delta$ is built up of terms in powers of $\mathrm{A}=Y_{u} Y_{u}^{\dagger}$ and $\mathrm{B}=Y_{d} Y_{d}^{\dagger}$, which transform as $(1 \oplus 8,1,1)$ under $G_{q}$.

Formally $\Delta$ comprises an infinite number of terms, namely $\Delta=\sum \xi_{j k l} \ldots \mathrm{A}^{j} \mathrm{~B}^{k} \mathrm{~A}^{l} \ldots$ with coefficients $\xi_{j k l}$.. expected to be at most of $\mathcal{O}(1)$. The MFV hypothesis requires that $\xi_{j k l . .}$ be real because complex $\xi_{j k l \ldots .}$ would introduce new $C P$-violation sources beyond that in the Yukawa couplings. Using the Cayley-Hamilton identity

$$
X^{3}=X^{2} \operatorname{Tr} X+\frac{1}{2} X\left[\operatorname{Tr} X^{2}-(\operatorname{Tr} X)^{2}\right]+\mathbb{1} \operatorname{Det} X
$$

\footnotetext{
${ }^{1}$ An analogous situation occurs in the type-III seesaw model [33].
} 
for an invertible $3 \times 3$ matrix $X$, one can resum the infinite series into a finite number of terms $[34,35]$

$$
\begin{aligned}
\Delta= & \xi_{1} \mathbb{1}+\xi_{2} \mathrm{~A}+\xi_{3} \mathrm{~B}+\xi_{4} \mathrm{~A}^{2}+\xi_{5} \mathrm{~B}^{2}+\xi_{6} \mathrm{AB}+\xi_{7} \mathrm{BA}+\xi_{8} \mathrm{ABA}+\xi_{9} \mathrm{BA}^{2} \\
& +\xi_{10} \mathrm{BAB}+\xi_{11} \mathrm{AB}^{2}+\xi_{12} \mathrm{ABA}^{2}+\xi_{13} \mathrm{~A}^{2} \mathrm{~B}^{2}+\xi_{14} \mathrm{~B}^{2} \mathrm{~A}^{2}+\xi_{15} \mathrm{~B}^{2} \mathrm{AB} \\
& +\xi_{16} \mathrm{AB}^{2} \mathrm{~A}^{2}+\xi_{17} \mathrm{~B}^{2} \mathrm{~A}^{2} \mathrm{~B}
\end{aligned}
$$

where $\mathbb{1}$ denotes a $3 \times 3$ unit matrix. One can then also utilize this to devise Hermitian combinations such as $\Delta_{q}=\Delta+\Delta^{\dagger}$.

Even though one starts with all $\xi_{j k l \ldots}$ being real, the resummation process will render the coefficients $\xi_{r}$ in eq. (2.5) generally complex due to imaginary parts generated among the traces of the matrix products $\mathrm{A}^{j} \mathrm{~B}^{k} \mathrm{~A}^{l} \cdots$ with $j+k+l+\cdots \geq 6$ upon the application of the Cayley-Hamilton identity. In appendix A we show the detailed reduction of one of the lowest-order products which give rise to the imaginary components of $\xi_{r}$. We find that the imaginary contributions are always reducible to factors proportional to $\operatorname{Im} \operatorname{Tr}\left(\mathrm{A}^{2} B A B^{2}\right)=$ $(i / 2) \operatorname{Det}[\mathrm{A}, \mathrm{B}]$ which is a Jarlskog invariant and much smaller than one [34].

Taking advantage of the invariance under $G_{q}$, we will work in the basis where $Y_{d}$ is diagonal,

$$
Y_{d}=\frac{\sqrt{2}}{v} \operatorname{diag}\left(m_{d}, m_{s}, m_{b}\right),
$$

and the fields $U_{k, L}, U_{k, R}, D_{k, L}$, and $D_{k, R}$ belong to the mass eigenstates. Hence we can write $Q_{k, L}$ and $Y_{u}$ in terms of the Cabibbo-Kobayashi-Maskawa (CKM) quark mixing matrix $V_{\mathrm{CKM}}$ as

$$
Q_{k, L}=\left(\begin{array}{c}
\left(V_{\mathrm{CKM}}^{\dagger}\right)_{k l} U_{l, L} \\
D_{k, L}
\end{array}\right), \quad Y_{u}=\frac{\sqrt{2}}{v} V_{\mathrm{CKM}}^{\dagger} \operatorname{diag}\left(m_{u}, m_{c}, m_{t}\right),
$$

where in the standard parametrization [9]

$$
V_{\mathrm{CKM}}=\left(\begin{array}{ccc}
c_{12} c_{13} & s_{12} c_{13} & s_{13} e^{-i \delta} \\
-s_{12} c_{23}-c_{12} s_{23} s_{13} e^{i \delta} & c_{12} c_{23}-s_{12} s_{23} s_{13} e^{i \delta} & s_{23} c_{13} \\
s_{12} s_{23}-c_{12} c_{23} s_{13} e^{i \delta} & -c_{12} s_{23}-s_{12} c_{23} s_{13} e^{i \delta} & c_{23} c_{13}
\end{array}\right),
$$

with $\delta$ being the $C P$ violation phase, $c_{k l}=\cos \theta_{k l}$, and $s_{k l}=\sin \theta_{k l}$. We note that, as a consequence, $\Delta_{u 8}$ and $\Delta_{d 8}$, whose basic building blocks are $Y_{u}^{\dagger} Y_{u}$ and $Y_{d}^{\dagger} Y_{d}$, respectively, are all diagonal and thus will not bring about new flavor- and $C P$-violating interactions.

For the lepton sector, since it is still unknown whether light neutrinos are Dirac or Majorana particles, we address the two possibilities separately. In the Dirac case, the $M_{\nu}$ part is absent from $\mathcal{L}_{\mathrm{m}}$ in eq. (2.1), which is therefore, in the MFV language, formally invariant under the global group $\mathrm{U}(3)_{L} \times \mathrm{U}(3)_{\nu} \times \mathrm{U}(3)_{E}=G_{\ell} \times \mathrm{U}(1)_{L} \times \mathrm{U}(1)_{\nu} \times \mathrm{U}(1)_{E}$ with $G_{\ell}=\mathrm{SU}(3)_{L} \times \mathrm{SU}(3)_{\nu} \times \mathrm{SU}(3)_{E}$. This means that the three generations of $L_{k, L}$, $\nu_{k, R}$, and $E_{k, R}$ transform as fundamental representations of $\mathrm{SU}(3)_{L, \nu, E}$ in $G_{\ell}$, respectively,

$$
L_{L} \rightarrow V_{L} L_{L}, \quad \nu_{R} \rightarrow V_{\nu} \nu_{R}, \quad E_{R} \rightarrow V_{E} E_{R}
$$


where $V_{L, \nu, E} \in \mathrm{SU}(3)$, whereas the Yukawa couplings are spurions transforming according to

$$
Y_{\nu} \rightarrow V_{L} Y_{\nu} V_{\nu}^{\dagger}, \quad Y_{e} \rightarrow V_{L} Y_{e} V_{E}^{\dagger}
$$

We will work in the basis where $Y_{e}$ is already diagonal,

$$
Y_{e}=\frac{\sqrt{2}}{v} \operatorname{diag}\left(m_{e}, m_{\mu}, m_{\tau}\right)
$$

and the fields $\nu_{k, L}, \nu_{k, R}, E_{k, L}$, and $E_{k, R}$ refer to the mass eigenstates. We can then express $L_{k, L}$ and $Y_{\nu}$ in terms of the Pontecorvo-Maki-Nakagawa-Sakata (PMNS) neutrino mixing matrix $U_{\mathrm{PMNS}}$ as

$$
L_{k, L}=\left(\begin{array}{c}
\left(U_{\mathrm{PMNS}}\right)_{k l} \nu_{l, L} \\
E_{k, L}
\end{array}\right), \quad Y_{\nu}=\frac{\sqrt{2}}{v} U_{\mathrm{PMNS}} \hat{m}_{\nu}, \quad \hat{m}_{\nu}=\operatorname{diag}\left(m_{1}, m_{2}, m_{3}\right),
$$

where $m_{1,2,3}$ are the light neutrino eigenmasses and $U_{\mathrm{PMNS}}$ has the same standard parametrization as in eq. (2.8). Thus the discussion for the down-type quarks can be easily applied to the charged leptons by replacing $V_{\mathrm{CKM}}$ with $U_{\mathrm{PMNS}}^{\dagger}$ and employing the building blocks $\mathrm{A}=Y_{\nu} Y_{\nu}^{\dagger}$ and $\mathrm{B}=Y_{e} Y_{e}^{\dagger}$ to construct $\Delta_{\nu}$ and $\Delta_{e}$, which are the lepton counterparts of $\Delta_{u}$ and $\Delta_{d}$, respectively.

If neutrinos are of Majorana nature, the $M_{\nu}$ part in eq. (2.1) is allowed. As a consequence, for $M_{\nu} \gg M_{\mathrm{D}}=v Y_{\nu} / \sqrt{2}$ the seesaw mechanism [24-32] becomes operational involving the $6 \times 6$ neutrino mass matrix

$$
\mathrm{M}=\left(\begin{array}{cc}
0 & M_{\mathrm{D}} \\
M_{\mathrm{D}}^{\mathrm{T}} & M_{\nu}
\end{array}\right)
$$

in the $\left(U_{\mathrm{PMNS}}^{*} \nu_{L}^{\mathrm{c}}, \nu_{R}\right)^{\mathrm{T}}$ basis. The resulting matrix of light neutrino masses is

$$
m_{\nu}=-\frac{v^{2}}{2} Y_{\nu} M_{\nu}^{-1} Y_{\nu}^{\mathrm{T}}=U_{\mathrm{PMNS}} \hat{m}_{\nu} U_{\mathrm{PMNS}}^{\mathrm{T}}
$$

where now $U_{\mathrm{PMNS}}$ contains the diagonal matrix $P=\operatorname{diag}\left(e^{i \alpha_{1} / 2}, e^{i \alpha_{2} / 2}, 1\right)$ multiplied from the right, $\alpha_{1,2}$ being the Majorana phases. It follows that $Y_{\nu}$ in eq. (2.12) is no longer valid, and one can instead take $Y_{\nu}$ to be [36]

$$
Y_{\nu}=\frac{i \sqrt{2}}{v} U_{\mathrm{PMNS}} \hat{m}_{\nu}^{1 / 2} O M_{\nu}^{1 / 2}
$$

where $O$ is a matrix satisfying $O O^{\mathrm{T}}=\mathbb{1}$ and $M_{\nu}=\operatorname{diag}\left(M_{1}, M_{2}, M_{3}\right)$. As we will see later, $O$ can provide a potentially important new source of $C P$ violation besides $U_{\mathrm{PMNS}}$. We comment that the presence of $M_{\nu}$ breaks the global $\mathrm{U}(3)_{\nu}$ completely if $M_{1,2,3}$ are unequal and partially into $\mathrm{O}(3)_{\nu}$ if $M_{1,2,3}$ are equal [22]. 


\section{Fermion EDMs in MFV framework}

To explore the MFV contribution to the EDMs of quarks and charged leptons, one needs to construct the relevant operators using $\Delta_{u, d, e}$ in combination with the quark, lepton, Higgs, and gauge fields. At leading order, the operators can be written as [21, 22]

$$
\begin{array}{ll}
O_{R L}^{(u 1)}=g^{\prime} \bar{U}_{R} Y_{u}^{\dagger} \Delta_{q u 1} \sigma_{\kappa \omega} \tilde{H}^{\dagger} Q_{L} B^{\kappa \omega}, & O_{R L}^{(u 2)}=g \bar{U}_{R} Y_{u}^{\dagger} \Delta_{q u 2} \sigma_{\kappa \omega} \tilde{H}^{\dagger} \tau_{a} Q_{L} W_{a}^{\kappa \omega} \\
O_{R L}^{(d 1)}=g^{\prime} \bar{D}_{R} Y_{d}^{\dagger} \Delta_{q d 1} \sigma_{\kappa \omega} H^{\dagger} Q_{L} B^{\kappa \omega}, & O_{R L}^{(d 2)}=g \bar{D}_{R} Y_{d}^{\dagger} \Delta_{q d 2} \sigma_{\kappa \omega} H^{\dagger} \tau_{a} Q_{L} W_{a}^{\kappa \omega} \\
O_{R L}^{(e 1)}=g^{\prime} \bar{E}_{R} Y_{e}^{\dagger} \Delta_{\ell 1} \sigma_{\kappa \omega} H^{\dagger} L_{L} B^{\kappa \omega}, & O_{R L}^{(e 2)}=g \bar{E}_{R} Y_{e}^{\dagger} \Delta_{\ell 2} \sigma_{\kappa \omega} H^{\dagger} \tau_{a} L_{L} W_{a}^{\kappa \omega}
\end{array}
$$

where $W$ and $B$ denote the usual $\mathrm{SU}(2)_{L} \times \mathrm{U}(1)_{Y}$ gauge fields with coupling constants $g$ and $g^{\prime}$, respectively, $\tau_{a}$ are Pauli matrices, $a=1,2,3$ is summed over, and $\Delta_{q u \varsigma, q d \varsigma, \ell \varsigma}$ with $\varsigma=1,2$ have the same form as $\Delta$ in eq. (2.5), but generally different $\xi_{r}$. One can express the effective Lagrangian containing these operators as

$$
\mathcal{L}_{\text {eff }}=\frac{1}{\Lambda^{2}}\left(O_{R L}^{(u 1)}+O_{R L}^{(u 2)}+O_{R L}^{(d 1)}+O_{R L}^{(d 2)}+O_{R L}^{(e 1)}+O_{R L}^{(e 2)}\right)+\text { H.c. },
$$

where $\Lambda$ is the MFV scale. In general the operators in $\mathcal{L}_{\text {eff }}$ have their own coefficients which have been absorbed by $\xi_{r}$ in their respective $\Delta$ 's. These coefficients also take into account the possibility that the MFV scale in the quark sector may differ from that in the lepton sector.

Expanding eq. (3.3), one can identify the terms relevant to fermion EDMs. In the quark sector the resulting EDMs of up- and down-type quarks are, respectively, proportional to $\operatorname{Im}\left(Y_{u}^{\dagger} \Delta_{q u \varsigma} V_{\mathrm{CKM}}^{\dagger}\right)_{k k}$ and $\operatorname{Im}\left(Y_{d}^{\dagger} \Delta_{q d \varsigma}\right)_{k k}$. The contributions of $\Delta_{q u \varsigma, q d \varsigma}$ to the EDMs come not only from some of the products of the $A$ and $B$ matrices therein, but also from the imaginary parts of $\xi_{r}$. As mentioned earlier, $\operatorname{Im} \xi_{r}$ are always proportional to $J_{\xi} \equiv$ $\operatorname{Im} \operatorname{Tr}\left(\mathrm{A}^{2} \mathrm{BAB}^{2}\right)=(i / 2) \operatorname{Det}[\mathrm{A}, \mathrm{B}]$, or explicitly

$$
J_{\xi}=\frac{-64\left(m_{u}^{2}-m_{c}^{2}\right)\left(m_{c}^{2}-m_{t}^{2}\right)\left(m_{t}^{2}-m_{u}^{2}\right)\left(m_{d}^{2}-m_{s}^{2}\right)\left(m_{s}^{2}-m_{b}^{2}\right)\left(m_{b}^{2}-m_{d}^{2}\right)}{v^{12}} J_{q},
$$

where $J_{q}=\operatorname{Im}\left(V_{u s} V_{c b} V_{u b}^{*} V_{c s}^{*}\right)=c_{12} s_{12} c_{23} s_{23} c_{13}^{2} s_{13} \sin \delta$ is a Jarlskog parameter for $V_{\mathrm{CKM}}$.

Not all of the products of $\mathrm{A}=Y_{u} Y_{u}^{\dagger}$ and $\mathrm{B}=Y_{d} Y_{d}^{\dagger}$ in $\Delta_{q u \varsigma, q d \varsigma}$ will contribute to quark EDMs. Since $Y_{u}$ has the form in eq. (2.7) and $Y_{d}$ is diagonal, the Hermiticity of $A$ and $B$ implies that only certain combinations of them are relevant. For example, $\left(Y_{d}^{\dagger} \mathrm{A}\right)_{k k}=\sqrt{2} m_{D_{k}} \mathrm{~A}_{k k} / v$ is purely real and hence does not affect $d_{D_{k}}$. In this case, one needs to have terms in $\Delta_{q d s}$ which are not Hermitian in order to have imaginary components in $\left(Y_{d}^{\dagger} \Delta_{q d \varsigma}\right)_{k k}$. We find that only two terms, proportional to $\mathrm{B}^{2} \mathrm{AB}$ and $\mathrm{B}^{2} \mathrm{~A}^{2} \mathrm{~B}$, are pertinent to the up-type quarks' EDMs and only the $A B A^{2}$ and $A B^{2} A^{2}$ terms are pertinent to the EDM's of down-type quarks.

The preceding discussions show that the contributions of $\operatorname{Im} \xi_{r}$ to the EDM of, say, the $u(d)$ quark are suppressed by a factor of $m_{c}^{2} / v^{2}\left(m_{s}^{2} m_{b}^{2} / v^{4}\right)$ compared to the contributions from $\mathrm{B}^{2} \mathrm{AB}\left(\mathrm{ABA}^{2}\right)$, which has the least number of suppressive factor from $Y_{u}\left(Y_{d}\right)$ among 
the products in eq. (2.5) that can potentially contribute. Hence we can neglect the impact of $\operatorname{Im} \xi_{r}$ on the quark EDMs. One, however, needs to take $\operatorname{Im} \xi_{r}$ into account when considering how MFV affects the contribution of the strong theta-term to the neutron EDM, as we demonstrate later.

Simplifying things, we arrive at the leading-order contributions to the $u$ - and $d$-quarks' EDMs

$$
\begin{aligned}
d_{u} & =\frac{\sqrt{2} e v}{\Lambda^{2}} \operatorname{Im}\left[Y_{u}^{\dagger}\left(\Delta_{q u 1}+\Delta_{q u 2}\right) V_{\mathrm{CKM}}^{\dagger}\right]_{11} \\
& =\frac{32 e m_{u}}{\Lambda^{2}}\left[\xi_{15}^{u}+\frac{2\left(m_{c}^{2}+m_{t}^{2}\right)}{v^{2}} \xi_{17}^{u}\right] \frac{\left(m_{c}^{2}-m_{t}^{2}\right)\left(m_{d}^{2}-m_{s}^{2}\right)\left(m_{s}^{2}-m_{b}^{2}\right)\left(m_{d}^{2}-m_{b}^{2}\right)}{v^{8}} J_{q}, \\
d_{d} & =\frac{\sqrt{2} e v}{\Lambda^{2}} \operatorname{Im}\left[Y_{d}^{\dagger}\left(\Delta_{q d 1}-\Delta_{q d 2}\right)\right]_{11} \\
& =\frac{32 e m_{d}}{\Lambda^{2}}\left[\xi_{12}^{d}+\frac{2\left(m_{s}^{2}+m_{b}^{2}\right)}{v^{2}} \xi_{16}^{d}\right] \frac{\left(m_{s}^{2}-m_{b}^{2}\right)\left(m_{u}^{2}-m_{c}^{2}\right)\left(m_{c}^{2}-m_{t}^{2}\right)\left(m_{u}^{2}-m_{t}^{2}\right)}{v^{8}} J_{q},
\end{aligned}
$$

where $\xi_{r}^{u}=\xi_{r}^{u 1}+\xi_{r}^{u 2}$ and $\xi_{r}^{d}=\xi_{r}^{d 1}-\xi_{r}^{d 2}$. The expressions for $d_{c, t}$ and $d_{s, b}$ can be simply derived from eqs. (3.5) and (3.6), respectively, by cyclically changing the quark labels. ${ }^{2}$

In the lepton sector, we get from eq. (3.3) the electron EDM

$$
\begin{aligned}
d_{e} & =\frac{\sqrt{2} e v}{\Lambda^{2}} \operatorname{Im}\left(Y_{e}^{\dagger} \Delta_{\ell 1}-Y_{e}^{\dagger} \Delta_{\ell 2}\right)_{11} \\
& =\frac{\sqrt{2} e v}{\Lambda^{2}}\left[\xi_{12}^{\ell} \operatorname{Im}\left(Y_{e}^{\dagger} \mathrm{ABA}^{2}\right)_{11}+\xi_{16}^{\ell} \operatorname{Im}\left(Y_{e}^{\dagger} \mathrm{AB}^{2} \mathrm{~A}^{2}\right)_{11}\right]
\end{aligned}
$$

where $\xi_{r}^{\ell}=\xi_{r}^{\ell 1}-\xi_{r}^{\ell 2}$, we have ignored $\operatorname{Im} \xi_{r}^{\ell}$, and here $\mathrm{A}=Y_{\nu} Y_{\nu}^{\dagger}$ and $\mathrm{B}=Y_{e} Y_{e}^{\dagger}$. If neutrinos are Dirac particles, analogously to $d_{d}$, we obtain

$d_{e}^{\mathrm{D}}=\frac{32 e m_{e}}{\Lambda^{2}}\left[\xi_{12}^{\ell}+\frac{2\left(m_{\mu}^{2}+m_{\tau}^{2}\right)}{v^{2}} \xi_{16}^{\ell}\right] \frac{\left(m_{\mu}^{2}-m_{\tau}^{2}\right)\left(m_{1}^{2}-m_{2}^{2}\right)\left(m_{2}^{2}-m_{3}^{2}\right)\left(m_{3}^{2}-m_{1}^{2}\right)}{v^{8}} J_{\ell}$,

where $J_{\ell}=\operatorname{Im}\left(U_{e 2} U_{\mu 3} U_{e 3}^{*} U_{\mu 2}^{*}\right)$ is a Jarlskog invariant for $U_{\mathrm{PMNS}}$.

In the case of Majorana neutrinos, if $\nu_{k, R}$ are degenerate, $M_{\nu}=\mathcal{M} \mathbb{1}$, and $O$ is a real orthogonal matrix, ${ }^{3}$ from eq. (2.15) we have

$$
\mathrm{A}=\frac{2}{v^{2}} \mathcal{M} U_{\mathrm{PMNS}} \hat{m}_{\nu} U_{\mathrm{PMNS}}^{\dagger}
$$

and consequently

$$
d_{e}^{\mathrm{M}}=\frac{32 e m_{e} \mathcal{M}^{3}}{\Lambda^{2} v^{8}}\left(m_{\mu}^{2}-m_{\tau}^{2}\right)\left(m_{1}-m_{2}\right)\left(m_{2}-m_{3}\right)\left(m_{3}-m_{1}\right) \xi_{12}^{\ell} J_{\ell},
$$

\footnotetext{
${ }^{2}$ It is worth commenting that $\operatorname{since} \operatorname{Im} \xi_{r} \propto \operatorname{Det}[\mathrm{A}, \mathrm{B}]$, due to the reality of the coefficients $\xi_{j k l \ldots}$ in the infinite series expansion of $\Delta$, and since $\mathrm{A}$ and $\mathrm{B}$ are Hermitian, $d_{q}$ would be identically zero if there were only one generation of fermions. The same applies to the lepton sector.

${ }^{3}$ Since the lepton Lagrangian with $\nu_{k, R}$ being degenerate is $\mathrm{O}(3)_{\nu}$ symmetric, one could transform this real $O$ into a unit matrix [37].
} 
the $\xi_{16}^{\ell}$ term having been neglected. Since $m_{k} \ll \mathcal{M}$, we can see that $d_{e}^{\mathrm{D}}$ is highly suppressed relative to $d_{e}^{\mathrm{M}}$. The formulas for $d_{\mu, \tau}^{\mathrm{D}}$ and $d_{\mu, \tau}^{\mathrm{M}}$ can be readily found from eqs. (3.8) and (3.10), respectively, by cyclically changing the mass subscripts.

In the discussion above, $d_{e}$ arises from the $C P$-violating Dirac phase $\delta$ in $U_{\mathrm{PMNS}}$, and the Majorana phases $\alpha_{1,2}$ therein do not participate. However, if $\nu_{k, R}$ are not degenerate, nonzero $\alpha_{1,2}$ can bring about an additional effect on $d_{e}$, even with a real $O \neq \mathbb{1}$. With a complex $O$, the phases in it may give rise to an extra contribution to $d_{e}$, whether or not $\nu_{k, R}$ are degenerate. The formulas for $d_{e}$ in these scenarios are more complicated than eq. (3.10) and are not shown here, but we will explore some of them numerically in the next section.

The various contributions to the fermion EDMs that we have considered have high powers in Yukawa couplings. Since the MFV hypothesis presupposes that all $C P$-violation effects originate from the Yukawa couplings, the high orders in them reflect the fact that nonvanishing EDMs in the SM begin to appear at the three-loop level for quarks and in higher loops for the electron. One may wonder whether these are the only contributions to fermion EDMs under the MFV framework. The answer is no because one can realize fermion EDMs by combining some lower-order Yukawa terms from the MFV operators with SM loop diagrams, such as those contributing to quark EDMs in the SM. Nevertheless, hereafter we will not include such type of possible contributions. The contributions that we have already covered should provide a good idea about how fermion EDMs are generated in the presence of MFV. For definiteness, we will apply numerically the results we have acquired and discuss some of their implications.

\section{Numerical analysis}

We will first treat the neutron EDM, $d_{n}$, evaluated from the quark contributions and infer from its data a bound on the scale of quark MFV. We will also look at how MFV affects the contribution of the strong $\theta$-term to $d_{n}$. Proceeding to the lepton sector, we will devote much of the section to the electron EDM, and briefly deal with the muon and tau EDMs, in order to explore limitations on the scale of leptonic MFV. Afterwards, we will examine constraints from $C P$-violating electron-nucleon interactions which were probed by recent searches for atomic and molecular EDMs. Finally, we will address potential restrictions from some $C P$-conserving processes.

\subsection{Neutron EDM}

In calculating quark EDMs, as in eqs. (3.5) and (3.6), one needs to take into account the running of the quark masses due to QCD evolution. We adopt the mass ranges $m_{u}=0.00139_{-0.00041}^{+0.0042}, \quad m_{d}=0.00285_{-0.00048}^{+0.00049}, m_{s}=0.058_{-0.012}^{+0.018}, \quad m_{c}=0.645_{-0.085}^{+0.043}$, $m_{b}=2.90_{-0.06}^{+0.16}$, and $m_{t}=174.2 \pm 1.2$, all in $\mathrm{GeV}$, at a renormalization scale $\mu=m_{W}$ from ref. [38]. With the central values of these masses and the quark Jarlskog parameter 
$J_{q}=\left(3.02_{-0.19}^{+0.16}\right) \times 10^{-5}$ from the latest fit by CKMfitter [39], we arrive at

$$
\begin{aligned}
d_{u} & =\frac{1.4 \times 10^{-35} e \mathrm{~cm}}{\Lambda^{2} / \mathrm{GeV}^{2}}\left(\xi_{15}^{u}+\xi_{17}^{u}\right), \quad d_{d}=\frac{1.3 \times 10^{-29} e \mathrm{~cm}}{\Lambda^{2} / \mathrm{GeV}^{2}}\left(\xi_{12}^{d}+0.00028 \xi_{16}^{d}\right), \\
d_{s} & =\frac{-2.6 \times 10^{-28} e \mathrm{~cm}}{\Lambda^{2} / \mathrm{GeV}^{2}}\left(\xi_{12}^{d}+0.00028 \xi_{16}^{d}\right),
\end{aligned}
$$

where $\xi_{r}^{u}=\xi_{r}^{u 1}+\xi_{r}^{u 2}$ and $\xi_{r}^{d}=\xi_{r}^{d 1}-\xi_{r}^{d 2}$. Evidently, the $s$-quark effect may be dominant.

To determine the neutron EDM, one needs to connect it to the quark-level quantities. The relation between $d_{n}$ and $d_{u, d, s}$ can be parameterized as

$$
d_{n}=\eta_{n}\left(\rho_{n}^{u} d_{u}+\rho_{n}^{d} d_{d}+\rho_{n}^{s} d_{s}\right)
$$

where $\eta_{n}=0.4$ accounts for corrections due to the QCD evolution from $\mu=m_{W}$ down to the hadronic scale [40] and the values of the parameters $\rho_{n}^{u, d, s}$ depend on the model for the matrix elements $\left\langle n\left|\bar{q} \sigma^{\kappa \omega} q\right| n\right\rangle=\rho_{n}^{q} \bar{u}_{n} \sigma^{\kappa \omega} u_{n}$. For instance, in the constituent quark model $\rho_{n}^{d}=\frac{4}{3}=-4 \rho_{n}^{u}$ and $\rho_{n}^{s}=0$ [1], whereas in the parton quark model $\rho_{n}^{u}=-0.508, \rho_{n}^{d}=$ 0.746 , and $\rho_{n}^{s}=-0.226$ [5]. From the various models proposed in the literature $[1,5,41]$, we may conclude that

$$
-0.78 \leq \rho_{n}^{u} \leq-0.17, \quad 0.7 \leq \rho_{n}^{d} \leq 2.1, \quad-0.35 \leq \rho_{n}^{s} \leq 0
$$

In view of these numbers and eq. (4.1), we can ignore the $d_{u}$ and $\xi_{16}^{d}$ terms. Hence, taking the extreme values $\rho_{n}^{d}=2.1$ and $\rho_{n}^{s}=-0.35$, as well as scanning over the quark mass and $J_{q}$ ranges quoted above to maximize $d_{n}$, we get

$$
d_{n}=\frac{8.4 \times 10^{-29} e \mathrm{~cm}}{\Lambda^{2} / \mathrm{GeV}^{2}} \xi_{12}^{d}
$$

It is then interesting to note that $\Lambda /\left|\xi_{12}^{d}\right|^{1 / 2}=100 \mathrm{GeV}$ translates into $d_{n}=8.4 \times$ $10^{-33} \mathrm{ecm}$, which is roughly similar to the SM expectation $d_{n}^{\mathrm{SM}} \sim 10^{-32}-10^{-31} \mathrm{ecm}[10$ 13]. Comparing eq. (4.4) with the current data $\left|d_{n}\right|_{\exp }<2.9 \times 10^{-26} e \mathrm{~cm}$ at $90 \%$ CL [9], we extract

$$
\frac{\Lambda}{\left|\xi_{12}^{d}\right|^{1 / 2}}>0.054 \mathrm{GeV}
$$

which is not strict at all. Less extreme choices of $\rho_{n}^{d, s}$ would lead to even weaker bounds. We conclude that the present neutron-EDM limit cannot yield a useful restriction on $\Lambda$.

One can also look at the contributions of quark chromo-EDMs to the neutron EDM [1]. The relevant operators are obtainable from the MFV quark-EDM operators by replacing $W_{a}^{\mu \nu}$ and $\tau_{a}$ with the gluon field strength tensor $G_{c}^{\mu \nu}$ and the color SU(3) generators $\lambda_{c}$, respectively. The extracted constraints on $\Lambda$ are similar. 


\subsection{MFV contribution to strong theta term}

Besides the quark (chromo-)EDMs, another contributor to the neutron EDM is the theta term of QCD [42-44], which in the SM is given by [5]

$$
\mathcal{L}_{\bar{\theta}}=\frac{-\bar{\theta} g_{\mathrm{s}}^{2}}{32 \pi^{2}} \epsilon_{\kappa v \phi \omega} G_{c}^{\kappa v} G_{c}^{\phi \omega},
$$

where $\bar{\theta}=\theta+\arg \operatorname{Det}\left(Y_{u} Y_{d}\right)$ involves the bare $\theta$-parameter, $g_{\mathrm{s}}$ is the strong coupling constant, and $\epsilon_{0123}=+1$. The inclusion of MFV causes $\bar{\theta}$ to be modified to

$$
\bar{\theta}_{\mathrm{MFV}}=\theta+\arg \operatorname{Det}\left(\Delta_{q u}^{\dagger} Y_{u} \Delta_{q d}^{\dagger} Y_{d}\right)=\bar{\theta}+\arg \operatorname{Det} \Delta_{q u}^{\dagger}+\arg \operatorname{Det} \Delta_{q d}^{\dagger},
$$

where $\Delta_{q u, q d}$ have the same expression as $\Delta$ in eq. (2.5), but generally different coefficients $\xi_{r}$. Although the addition of these new factors to the Yukawa Lagrangian amounts only to a redefinition of $Y_{u, d}$ and hence has no direct experimental implications after the quark mass matrices are diagonalized, we can expect that $\Delta_{q u, q d}$ are close to the unit matrix. Our interest is in investigating the size of $\arg \operatorname{Det} \Delta_{q u, q d}$ in eq. (4.7) and thus whether or not their presence makes the fine tuning between the two terms in $\bar{\theta}$ worse.

To compute Det $\Delta_{q u}$, we first write the real and imaginary parts of $\xi_{r}$ in terms of real constants $\varrho_{r}$ and $\imath_{r}$ as

$$
\operatorname{Re} \xi_{r}=\varrho_{r}, \quad \operatorname{Im} \xi_{r}=\imath_{r} J_{\xi}
$$

with $J_{\xi}$ given in eq. (3.4). Upon applying the Cayley-Hamilton identity, we then get

$$
\operatorname{Det} \Delta_{q u}=\frac{1}{6}\left(\operatorname{Tr} \Delta_{q u}\right)^{3}-\frac{1}{2} \operatorname{Tr} \Delta_{q u} \operatorname{Tr}\left(\Delta_{q u}^{2}\right)+\frac{1}{3} \operatorname{Tr}\left(\Delta_{q u}^{3}\right),
$$

which leads us to

$$
\begin{aligned}
\operatorname{Re}\left(\operatorname{Det} \Delta_{q u}\right) \simeq & \varrho_{1}^{3}+\varrho_{1}^{2}\left(\varrho_{2} y_{t}^{2}+\varrho_{4} y_{t}^{4}\right) \\
J_{\xi}^{-1} \operatorname{Im}\left(\operatorname{Det} \Delta_{q u}\right) \simeq & -\varrho_{2}\left[\varrho_{2} \varrho_{15}+\varrho_{3}\left(\varrho_{13}-\varrho_{14}\right)+\varrho_{5} \varrho_{9}+\varrho_{7} \varrho_{11}\right]-\varrho_{3}\left(\varrho_{3} \varrho_{12}-\varrho_{4} \varrho_{11}-\varrho_{6} \varrho_{9}\right) \\
& -\left(\varrho_{6}-\varrho_{7}\right)\left(\varrho_{2} \varrho_{10}+\varrho_{3} \varrho_{8}+\varrho_{4} \varrho_{5}-\varrho_{6} \varrho_{7}\right)-\varrho_{2}\left(\varrho_{4} \varrho_{17}+\varrho_{9} \varrho_{13}\right) y_{t}^{4} \\
& +\left[-\varrho_{2}\left(\varrho_{2} \varrho_{17}+\varrho_{4} \varrho_{15}-\varrho_{6} \varrho_{14}+\varrho_{7} \varrho_{13}+\varrho_{9} \varrho_{11}\right)-\varrho_{3}\left(\varrho_{6} \varrho_{12}-\varrho_{8} \varrho_{9}\right)\right. \\
& \left.\quad-\left(\varrho_{6}-\varrho_{7}\right)\left(\varrho_{4} \varrho_{10}-\varrho_{6} \varrho_{9}\right)\right] y_{t}^{2} \\
+ & \varrho_{1}\left\{-\varrho_{2} \varrho_{17}-\varrho_{3} \varrho_{16}+\varrho_{4} \varrho_{15}+\varrho_{5} \varrho_{12}+\varrho_{6} \varrho_{13}-\varrho_{7} \varrho_{14}+\varrho_{8} \varrho_{11}-\varrho_{9} \varrho_{10}\right. \\
& \quad+\left[2 \varrho_{2} \imath_{1}-\varrho_{6} \varrho_{16}+\varrho_{8}\left(\varrho_{13}-\varrho_{14}\right)+\varrho_{11} \varrho_{12}\right] y_{t}^{2} \\
& \left.\quad+\left(2 \varrho_{4} \imath_{1}+\varrho_{12} \varrho_{13}\right) y_{t}^{4}\right\} \\
+ & \varrho_{1}^{2}\left(3 \iota_{1}+\imath_{2} y_{t}^{2}+\imath_{4} y_{t}^{4}\right),
\end{aligned}
$$

where $y_{q}=\sqrt{2} m_{q} / v$ and on the right-hand sides we have ignored terms suppressed by powers of $y_{u, c, d, s, b}$. The formulas for Det $\Delta_{q d}$ are similar.

Since $y_{t}^{2} \sim 1 \gg y_{u, c, d, s, b}^{2}$, the requirement that $\Delta_{q u, q d} \simeq \mathbb{1}$ implies that

$$
\varrho_{1} \simeq 1, \quad\left|\varrho_{2,4}\right| \ll 1, \quad\left|\varrho_{3,5,6, \ldots, 17}\right| \leq \mathcal{O}(1), \quad\left|\imath_{1,2, \ldots, 17}\right| \leq \mathcal{O}(1),
$$

Using these conditions and the quark parameter values employed earlier, we have checked numerically that eqs. (4.10) and (4.11) approximate well the exact (but much lengthier) expressions, especially if $\left|\varrho_{2,4}\right| \leq \mathcal{O}(0.001)$. Moreover, we get $\left|\arg \operatorname{Det} \Delta_{q u, q d}\right|<10^{-21}$. Obviously, the MFV effect is negligible compared to the present bound $\bar{\theta}_{\exp }<10^{-10}$ [5]. 


\begin{tabular}{|c|c|c|}
\hline Observable & $\mathrm{NH}$ & $\mathrm{IH}$ \\
\hline $\sin ^{2} \theta_{12}$ & $0.308 \pm 0.017$ & $0.308 \pm 0.017$ \\
$\sin ^{2} \theta_{23}$ & $0.425_{-0.027}^{+0.029}$ & $0.437_{-0.029}^{+0.059}$ \\
$\sin ^{2} \theta_{13}$ & $0.0234_{-0.0018}^{+0.0022}$ & $0.0239 \pm 0.0021$ \\
$\delta / \pi$ & $1.39_{-0.27}^{+0.33}$ & $1.35_{-0.39}^{+0.24}$ \\
$\Delta m_{21}^{2}=m_{2}^{2}-m_{1}^{2}$ & $\left(7.54_{-0.22}^{+0.26}\right) \times 10^{-5} \mathrm{eV}^{2}$ & $\left(7.54_{-0.22}^{+0.26}\right) \times 10^{-5} \mathrm{eV}^{2}$ \\
$\Delta m^{2}=\left|m_{3}^{2}-\left(m_{1}^{2}+m_{2}^{2}\right) / 2\right|$ & $\left(2.44_{-0.06}^{+0.08}\right) \times 10^{-3} \mathrm{eV}^{2}$ & $(2.40 \pm 0.07) \times 10^{-3} \mathrm{eV}^{2}$ \\
\hline
\end{tabular}

Table 1. Results of a recent fit to the global data on neutrino oscillations [45]. The neutrino mass hierarchy may be normal $\left(m_{1}<m_{2}<m_{3}\right)$ or inverted $\left(m_{3}<m_{1}<m_{2}\right)$.

\subsection{Electron EDM}

To evaluate the EDMs of charged leptons, we need the values of the various pertinent quantities, such as the elements of the neutrino mixing matrix $U_{\mathrm{PMNS}}$ as well as the masses of neutrinos and charged leptons. If neutrinos are Dirac in nature, the parametrization of $U_{\text {PMNS }}$ is the same as $V_{\text {CKM }}$ in eq. (2.8). In table 1 , we have listed $\sin ^{2} \theta_{k l}$ and $\delta$ from a recent fit to global neutrino data [45]. Most of these numbers depend on whether neutrino masses fall into a normal hierarchy (NH), where $m_{1}<m_{2}<m_{3}$, or an inverted one (IH), where $m_{3}<m_{1}<m_{2}$. If neutrinos are Majorana particles, $U_{\text {PMNS }}$ contains an additional matrix $P=\operatorname{diag}\left(e^{i \alpha_{1} / 2}, e^{i \alpha_{2} / 2}, 1\right)$ multiplied from the right, where $\alpha_{1,2}$ are the Majorana phases which remain unknown.

Also listed in table 1 are the differences in neutrinos' squared masses, which are well determined. In contrast, our knowledge about the absolute scale of the masses is still poor. Some information on the latter is available from tritium $\beta$-decay experiments [46, 47]. In particular, their latest results imply an upper limit on the (electron based) antineutrino mass of $m_{\bar{\nu}_{e}}<2 \mathrm{eV}$ [9]. Planned measurements will be more sensitive by an order of magnitude [46, 47]. Indirectly, stronger bounds on the total mass $\Sigma_{k} m_{k}=m_{1}+m_{2}+m_{3}$ can be inferred from cosmological observations. Specifically, the Planck Collaboration extracted $\Sigma_{k} m_{k}<0.23 \mathrm{eV}$ at $95 \% \mathrm{CL}$ from cosmic microwave background (CMB) and baryon acoustic oscillation (BAO) measurements [48]. Including additional observations can improve this limit to $\Sigma_{k} m_{k}<0.18 \mathrm{eV}$ [49]. On the other hand, there are also recent analyses that have turned up tentative indications of bigger masses and hence quasidegeneracy (QD) among the neutrinos. The South Pole Telescope Collaboration reported $\Sigma_{k} m_{k}=(0.32 \pm 0.11) \mathrm{eV}$ from the combined CMB, BAO, Hubble constant, and SunyaevZeldovich selected galaxy cluster abundances dataset [50]. This is compatible with the later finding $\Sigma_{k} m_{k}=(0.36 \pm 0.10) \mathrm{eV}$ favored by the Baryon Oscillation Spectroscopic Survey CMASS Data Release 11 [51]. In the following numerical work, we take this QD possibility into consideration.

If neutrinos are of Dirac nature, we first note that the mass difference definitions in table 1 imply that $\left(m_{1}^{2}-m_{2}^{2}\right)\left(m_{2}^{2}-m_{3}^{2}\right)\left(m_{3}^{2}-m_{1}^{2}\right)=\Delta m_{21}^{2}\left(\Delta m^{2}\right)^{2}-\frac{1}{4}\left(\Delta m_{21}^{2}\right)^{3}$, which is independent of $m_{k}$ individually. Then, scanning the parameter ranges in table 1 to 
maximize $d_{e}^{\mathrm{D}}$ in eq. (3.8), we obtain for the $\mathrm{NH}(\mathrm{IH})$ of neutrino masses [23]

$$
d_{e}^{\mathrm{D}}=\frac{1.3(1.3) \times 10^{-99} e \mathrm{~cm}}{\Lambda^{2} / \mathrm{GeV}^{2}} \xi_{12}^{\ell},
$$

after dropping the $\xi_{16}^{\ell}$ part. This is negligible compared to the latest data $\left|d_{e}\right|_{\exp }<$ $8.7 \times 10^{-29} e \mathrm{~cm}$ reported by ACME [7], and the smallness is due to the light neutrino masses being tiny.

In contrast, if neutrinos are Majorana particles, $d_{e}$ can be sizable. To see this, we begin with the simplest possibility that $\nu_{k, R}$ are degenerate, $M_{\nu}=\mathcal{M} \mathbb{1}$, and the $O$ matrix in eq. (2.15) is real. For this scenario, $d_{e}$ is already given in eq. (3.10), which depends on the choice for one of $m_{1,2,3}$ after the mass data are included. Scanning again the empirical parameter ranges in table 1 to maximize $d_{e}^{\mathrm{M}}$, we obtain for $m_{1}=0\left(m_{3}=0\right)$ in the $\mathrm{NH}$ (IH) case

$$
\frac{d_{e}^{\mathrm{M}}}{e \mathrm{~cm}}=4.7(0.52) \times 10^{-23}\left(\frac{\mathcal{M}}{10^{15} \mathrm{GeV}}\right)^{3}\left(\frac{\mathrm{GeV}}{\hat{\Lambda}}\right)^{2},
$$

where $\hat{\Lambda}=\Lambda /\left|\xi_{12}^{\ell}\right|^{1 / 2}$. Then $\left|d_{e}^{\exp }\right|<8.7 \times 10^{-29} e \mathrm{~cm}[7]$ implies

$$
\hat{\Lambda}>0.74(0.24) \mathrm{TeV}\left(\frac{\mathcal{M}}{10^{15} \mathrm{GeV}}\right)^{3 / 2} \text {. }
$$

Although this might suggest that $\hat{\Lambda}$ could be extremely high with an excessively large $\mathcal{M}$, there are limitations on $\mathcal{M}$. Since the series in eq. (2.5), which implicitly incorporates arbitrarily high powers of $A$ and $B$, has to converge, their eigenvalues need to be capped [23, 35]. Otherwise, the coefficients $\xi_{r}$ might not converge to finite numbers after the reduction of $\Delta$ from its infinite series expansion to eq. (2.5). In the lepton sector, we only need to be concerned with $\mathrm{A}=Y_{\nu} Y_{\nu}^{\dagger}$, as $\mathrm{B}=Y_{e} Y_{e}^{\dagger}$ already has diminished eigenvalues. Thus one may demand that the eigenvalues of $A$ are at most 1 . However, since MFV may emerge from calculations of SM loops, the expansion quantities may be more naturally be $A /\left(16 \pi^{2}\right)$ and $B /\left(16 \pi^{2}\right)$, in which case the maximum eigenvalue of $A$ cannot be more than $16 \pi^{2}$. As another alternative, one may impose the perturbativity condition on the Yukawa couplings, namely $\left(Y_{\nu}\right)_{j k}<\sqrt{4 \pi}[52]$, implying a cap of $4 \pi$ instead.

In this paper we require the eigenvalues of $\mathrm{A}=Y_{\nu} Y_{\nu}^{\dagger}$ not to exceed unity. Furthermore, in our illustrations we will choose the largest eigenmasses of the right-handed neutrinos subject to this condition. For the example resulting in eq. (4.15), this translates into the maximal value $\mathcal{M}=6.16(6.22) \times 10^{14} \mathrm{GeV}$ in the $\mathrm{NH}(\mathrm{IH})$ case and consequently

$$
\hat{\Lambda}>0.36(0.12) \mathrm{TeV} .
$$

This constraint would weaken if $m_{1(3)}>0$. For comparison with later illustrations, the $\mathcal{M}$ numbers above translate into $d_{e}^{\mathrm{M}} \hat{\Lambda}^{2}=1.1(0.13) \times 10^{-23} e \mathrm{~cm}$.

Now, with $\nu_{k, R}$ still degenerate, $M_{\nu}=\mathcal{M} \mathbb{1}$, but $O$ complex, A has a less simple expression,

$$
\mathrm{A}=\frac{2}{v^{2}} \mathcal{M} U_{\mathrm{PMNS}} \hat{m}_{\nu}^{1 / 2} O O^{\dagger} \hat{m}_{\nu}^{1 / 2} U_{\mathrm{PMNS}}^{\dagger}
$$


which is to be applied to $d_{e}^{\mathrm{M}}$ in eq. (3.7). From now on, we ignore the $\xi_{16}^{\ell}$ parts. We can always write $O O^{\dagger}=e^{2 i \mathrm{R}}$ with a real antisymmetric matrix

$$
\mathrm{R}=\left(\begin{array}{ccc}
0 & r_{1} & r_{2} \\
-r_{1} & 0 & r_{3} \\
-r_{2} & -r_{3} & 0
\end{array}\right)
$$

Since $O O^{\dagger}$ is not diagonal, A will in general have dependence on the Majorana phases in $U_{\text {PMNS }}$ if they are not zero. To concentrate first on demonstrating how $O$ can give rise to $C P$ violation beyond that induced by the Dirac phase $\delta$ in $U_{\mathrm{PMNS}}$, we switch off the Majorana phases, $\alpha_{1,2}=0$. Subsequently, for illustrations, we pick two possible sets of $r_{1,2,3}$, namely, (i) $r_{1}=-r_{2}=r_{3}=-\rho$ and (ii) $r_{1}=2 r_{2}=3 r_{3}=\rho$, and employ the central values of the data in table 1 , particularly

$$
\delta=1.39 \pi(\mathrm{NH}) \text { or } 1.35 \pi(\mathrm{IH}) \text {. }
$$

We present in figure $1(\mathrm{a})$-(d) the resulting $d_{e}^{\mathrm{M}} \hat{\Lambda}^{2}$ versus $\rho$ for the $\mathrm{NH}(\mathrm{IH})$ of light neutrino masses with $m_{1(3)}=0$. Since $\delta$ is not yet well-determined, we also depict the variations of $d_{e}^{\mathrm{M}}$ over the one-sigma ranges of $\delta$ quoted in table 1 with the lighter blue and red bands. We remark that the boundaries of the bands do not necessarily correspond to the upper or lower ends of the $\delta$ ranges. Within these bands, the blue and red solid curves belong, respectively, to the NH and IH central values in eq. (4.19). We also graph the (dashed) curves for $\delta=0$ to reveal the $C P$-violating role of $O$ alone. The solid and dashed curves in figure $1(\mathrm{a}, \mathrm{b})$ are roughly the mirror images about $\rho=0$ of the corresponding curves given in ref. [23] for $r_{1,2,3}=\rho$.

In figure 1(a)-(d), as well as in ref. [23], we have only examples where the lightest neutrinos are massless and, consequently, the neutrino masses sum up to $\Sigma_{k} m_{k}=0.059 \mathrm{eV}$ and $0.099 \mathrm{eV}$ in the $\mathrm{NH}$ and $\mathrm{IH}$ cases, respectively. These numbers satisfy the aforementioned bound from cosmological data, $\Sigma_{k} m_{k}<0.18 \mathrm{eV}$ [49]. In light of the hints of quasidegenerate neutrinos with $\Sigma_{k} m_{k} \sim 0.3 \mathrm{eV}$ from other cosmological observations [50, 51], which still need confirmation by future measurements, here we also provide a couple of instances in figure $1(\mathrm{e}, \mathrm{f})$ after making the NH choice $m_{1}=0.1 \mathrm{eV}<m_{2}<m_{3}$, which translates into $\Sigma_{k} m_{k}=0.31 \mathrm{eV}$.

All these examples in figure 1 clearly indicate that $O$ can generate potentially significant new effects of $C P$ violation which can exceed those of $\delta$. The latter point is most noticeable in figure $1(\mathrm{~b}, \mathrm{~d})$ from comparing the IH $\delta \neq 0$ regions at $\rho \sim 0$ with the extreme values of the corresponding IH $\delta=0$ curves.

With $\alpha_{1,2}=0$, the $C P$-violating impact of $O$ can still materialize even if it is real provided that $\nu_{k, R}$ are not degenerate. In that case

$$
\mathrm{A}=\frac{2}{v^{2}} U_{\mathrm{PMNS}} \hat{m}_{\nu}^{1 / 2} O M_{\nu} O^{\dagger} \hat{m}_{\nu}^{1 / 2} U_{\mathrm{PMNS}}^{\dagger}
$$

based on eq. (2.15). For instance, assuming that $O$ is real, $O=e^{\mathrm{R}}$ with $r_{1}=-r_{2}=r_{3}=$ $-\rho$, and that $M_{\nu}=\mathcal{M} \operatorname{diag}(1,0.8,1.2)$, we show the resulting $d_{e}^{\mathrm{M}} \hat{\Lambda}^{2}$ versus $\rho$ in figure 2(a), 

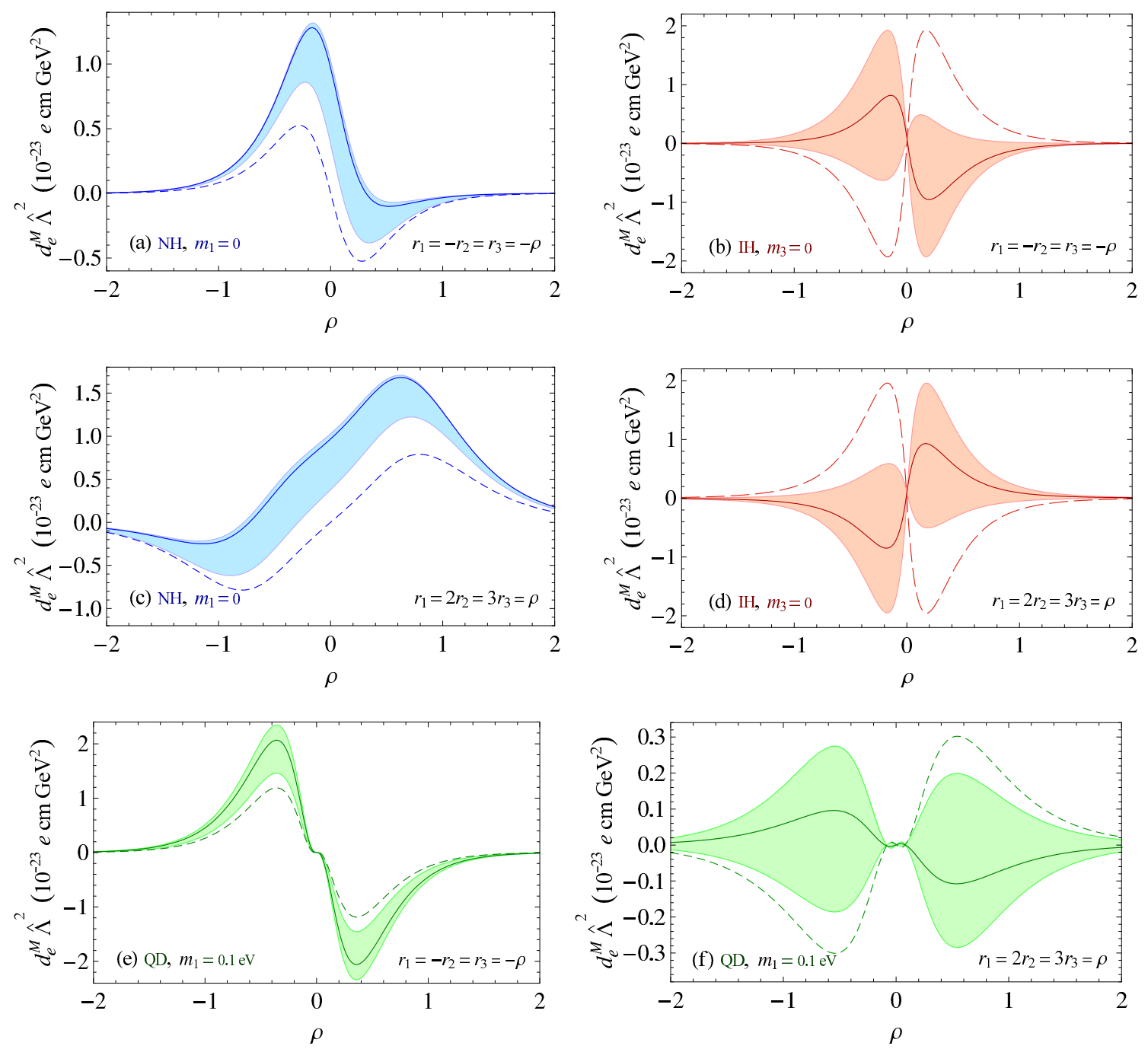

Figure 1. Dependence of $d_{e}^{\mathrm{M}}$ times $\hat{\Lambda}^{2}=\Lambda^{2} /\left|\xi_{12}^{\ell}\right|$ on the $O$-matrix parameter $\rho$ in the absence of the Majorana phases, $\alpha_{1,2}=0$, for degenerate $\nu_{k, R}$ and complex $O$ with (a,b,e) $r_{1}=-r_{2}=r_{3}=-\rho$ and (c,d,f) $r_{1}=2 r_{2}=3 r_{3}=\rho$, as explained in the text. The lighter blue, red, and green bands reflect the one-sigma ranges of $\delta$, while the solid and dashed curves correspond, respectively, to its central values in eq. (4.19) and to $\delta=0$. In (e,f) and other QD plots below, only the NH scenario is assumed, unless stated otherwise.

where only the $\delta \neq 0$ curves are nonvanishing and the sinusoidal behavior of $d_{e}$ is visible. As in the previous figure, we also display the variations of $d_{e}^{\mathrm{M}}$ over the one-sigma ranges of $\delta$ from table 1 . The solid curves in figure 2(a) are similar to their $r_{1,2,3}=\rho$ counterparts in ref. [23]. As another example, we select again $r_{1}=2 r_{2}=3 r_{3}=\rho$, keeping the other input parameters unchanged, and plot figure $2(\mathrm{~b})$ which differs somewhat qualitatively from figure 2(a). In figure 2(c,d) we graph the QD cases with $m_{1}=0.1 \mathrm{eV}<m_{2}<m_{3}$, which turn out to have much smaller $d_{e}^{\mathrm{M}}$ ranges. All of these results further demonstrate the importance of $O$ as an extra source of $C P$ violation.

Turning our attention now to the contribution of the Majorana phases, we first illustrate it for $M_{\nu}=\mathcal{M} \mathbb{1}$ and $O=e^{i \mathrm{R}}$ with the two sets of $r_{1,2,3}$ chosen in the previous 

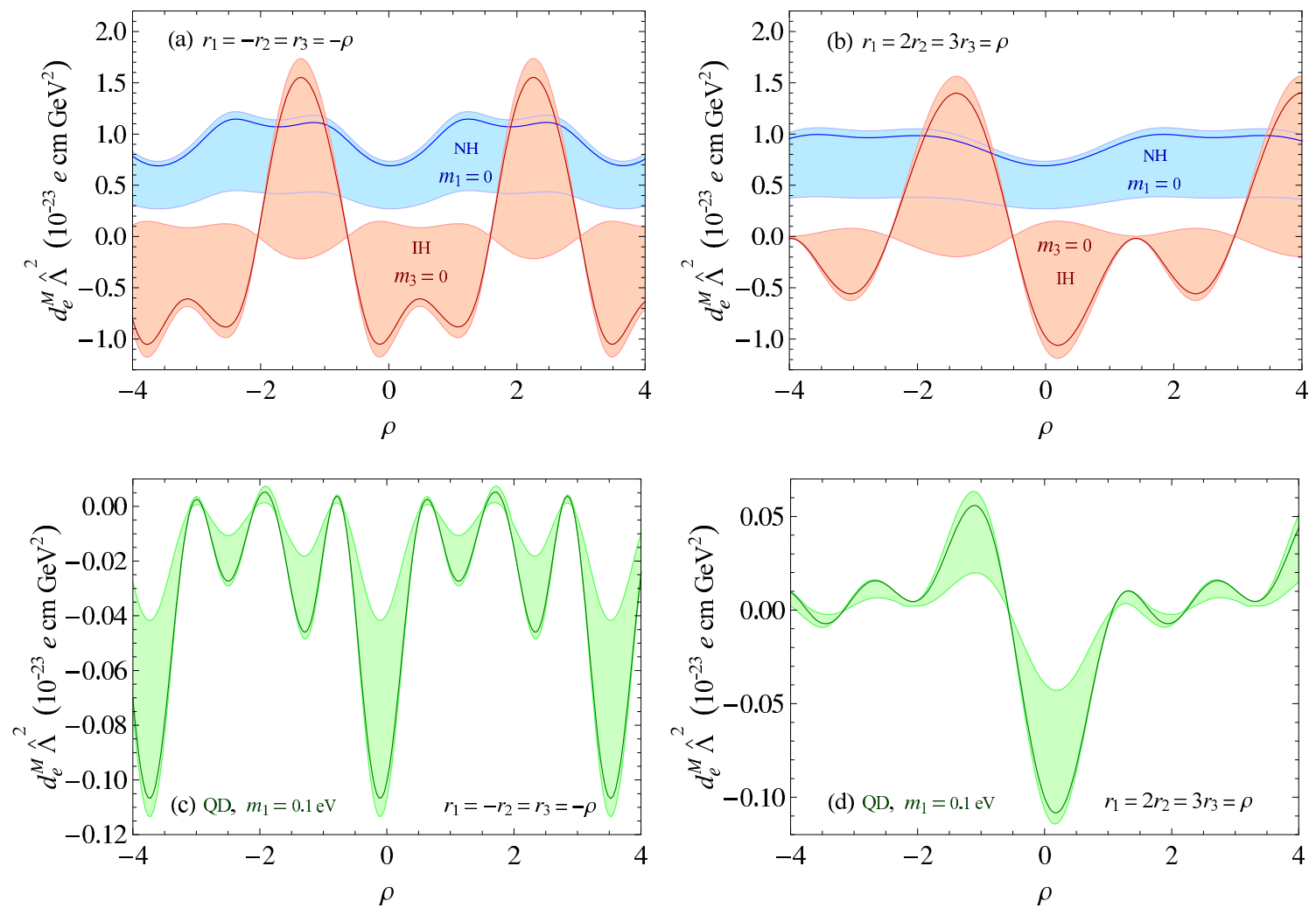

Figure 2. Dependence of $d_{e}^{\mathrm{M}} \hat{\Lambda}^{2}$ on $O$-matrix parameter $\rho$ in the absence of Majorana phases, $\alpha_{1,2}=0$, for nondegenerate $\nu_{k, R}$ with $M_{\nu}=\mathcal{M} \operatorname{diag}(1,0.8,1.2)$ and real $O=e^{\mathrm{R}}$ with $(\mathrm{a}, \mathrm{c}) r_{1}=$ $-r_{2}=r_{3}=-\rho$ and $(\mathrm{b}, \mathrm{d}) r_{1}=2 r_{2}=3 r_{3}=\rho$, as explained in the text. The lighter blue, red, and green bands reflect the one-sigma ranges of $\delta$, while the solid curves correspond to its central values in eq. (4.19).

paragraph. Thus, fixing $\alpha_{1}=0$ and $\rho=\frac{1}{2}$, we depict the resulting dependence of $d_{e}^{\mathrm{M}}$ on $\alpha_{2}$ in figure 3 for nonzero $\delta$ within its one-sigma ranges from table 1 and also for $\delta=0$. For further illustrations, we do the same with $M_{\nu}=\mathcal{M} \operatorname{diag}(1,0.8,1.2)$ and $O=e^{\mathrm{R}}$, displaying the results in figure 4. It is noticeable that each of the solid or dashed curves in figures 3 and 4 repeats itself after $\alpha_{2}$ changes by $4 \pi$, which is attributable to the $e^{i \alpha_{2} / 2}$ dependence of $d_{e}^{\mathrm{M}}$ in these cases. Also, one can verify visually that the solid curves in figures 1 and 3 (2 and 4) are consistent with each other at $\rho=\frac{1}{2}$ and $\alpha_{1,2}=0$. It is evident from the instances in figures 3 and 4 , as well as their counterparts in ref. [23], that the Majorana phases yield additional important $C P$-violating effects on $d_{e}$ beyond $\delta$.

It is interesting that some of the $C P$-violating variables which enter $d_{e}^{\mathrm{M}}$ also affect neutrinoless double- $\beta$ decay due to the Majorana nature of the electron neutrino. This process is of fundamental importance because it does not conserve lepton number and thus will be evidence for new physics if detected $[46,47]$. If there are no other contributions, the rate of neutrinoless double- $\beta$ decay increases with the square of the effective Majorana mass

$$
\begin{aligned}
\left\langle m_{\beta \beta}\right\rangle & =\left|\sum_{k} U_{e k}^{2} \hat{m}_{k}\right|=\left|\left(U_{\mathrm{PMNS}} \hat{m}_{\nu} U_{\mathrm{PMNS}}^{\mathrm{T}}\right)_{11}\right| \\
& =\left|c_{12}^{2} c_{13}^{2} m_{1} e^{i \alpha_{1}}+s_{12}^{2} c_{13}^{2} m_{2} e^{i \alpha_{2}}+s_{13}^{2} m_{3} e^{-2 i \delta}\right| .
\end{aligned}
$$



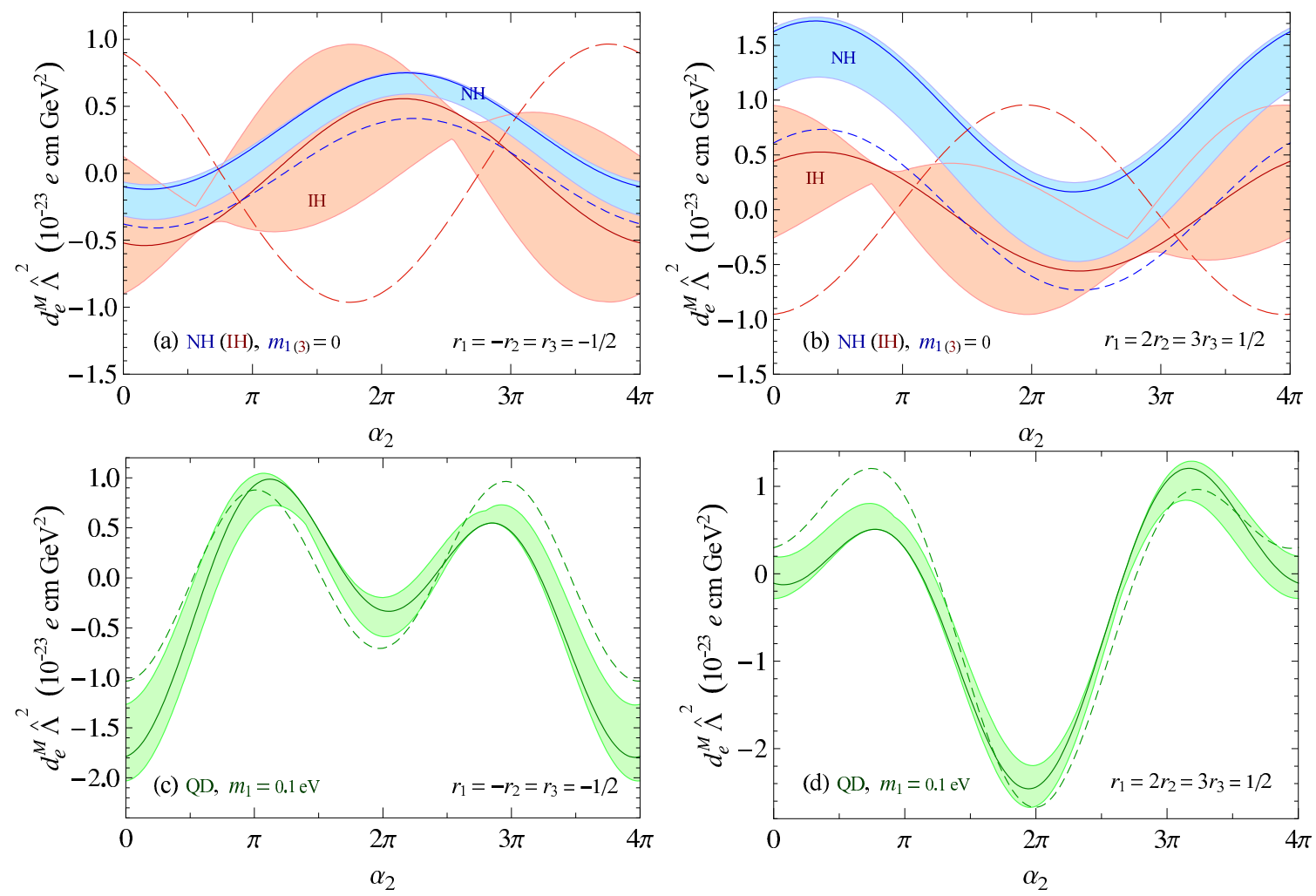

Figure 3. Dependence of $d_{e}^{\mathrm{M}} \hat{\Lambda}^{2}$ on $\alpha_{2}$ for $\alpha_{1}=0$, degenerate $\nu_{k, R}$, and $O=e^{i \mathrm{R}}$ with $(\mathrm{a}, \mathrm{c}) r_{1,3}=$ $-r_{2}=-\frac{1}{2}$ and $(\mathrm{b}, \mathrm{d}) r_{1}=2 r_{2}=3 r_{3}=\frac{1}{2}$, as explained in the text. The bands and curves have the same meanings as in preceding figures.

In figure 5 we display several examples of $\left\langle m_{\beta \beta}\right\rangle$ versus $\alpha_{2}$ for $\alpha_{1}=0$, but not those for $\delta=0$ to avoid crowding the plots. It is obvious that each of the curves repeats itself after $\alpha_{2}$ changes by $2 \pi$, which is due to the presence of $e^{i \alpha_{2}}$ in $\left\langle m_{\beta \beta}\right\rangle$, unlike the $d_{e}^{\mathrm{M}}$ curves in figures 3 and 4 . The peak values in the third plot of figure 5 are already close to the

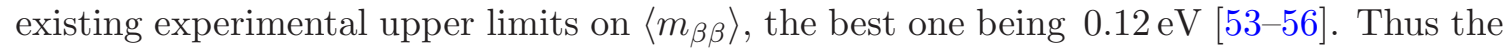
QD possibility will be tested by forthcoming searches within the next decade, which are expected to have sensitivities reaching $0.04 \mathrm{eV}$ to $0.01 \mathrm{eV}$ [57].

From figures $3-5$, one can conclude that $d_{e}^{\mathrm{M}}$ and $\left\langle m_{\beta \beta}\right\rangle$ may be correlated. For the MFV scenario under consideration and the parameter choices we made with the central values from table 1, we show in figure 6 some sample relations between the two observables. One can see in particular that the plots in figure $6(\mathrm{a}, \mathrm{c})[(\mathrm{d}, \mathrm{f})]$ are related to the solid curves in the first and third (green) graphs of figure 5, respectively, and the corresponding solid curves in figure 3 [figure 4] for $r_{1}=2 r_{2}=3 r_{3}=\frac{1}{2}$. In figure 6 we have also indicated a projected sensitivity of $0.04 \mathrm{eV}$ in future hunts for neutrinoless double- $\beta$ decay which may be achieved after several years.

The illustrations in figure 6 suggest that, if searches in coming years still yield null results, the acquired limits on $d_{e}$ and $\left\langle m_{\beta \beta}\right\rangle$ will impose significant restrictions on various scenarios based on lepton MFV. On the other hand, unambiguous observations of $d_{e}^{\mathrm{M}}$ and/or neutrinoless double-beta decay will help pin down the favored underlying model and parameter space, under the assumption that the latter process is mediated by a light 

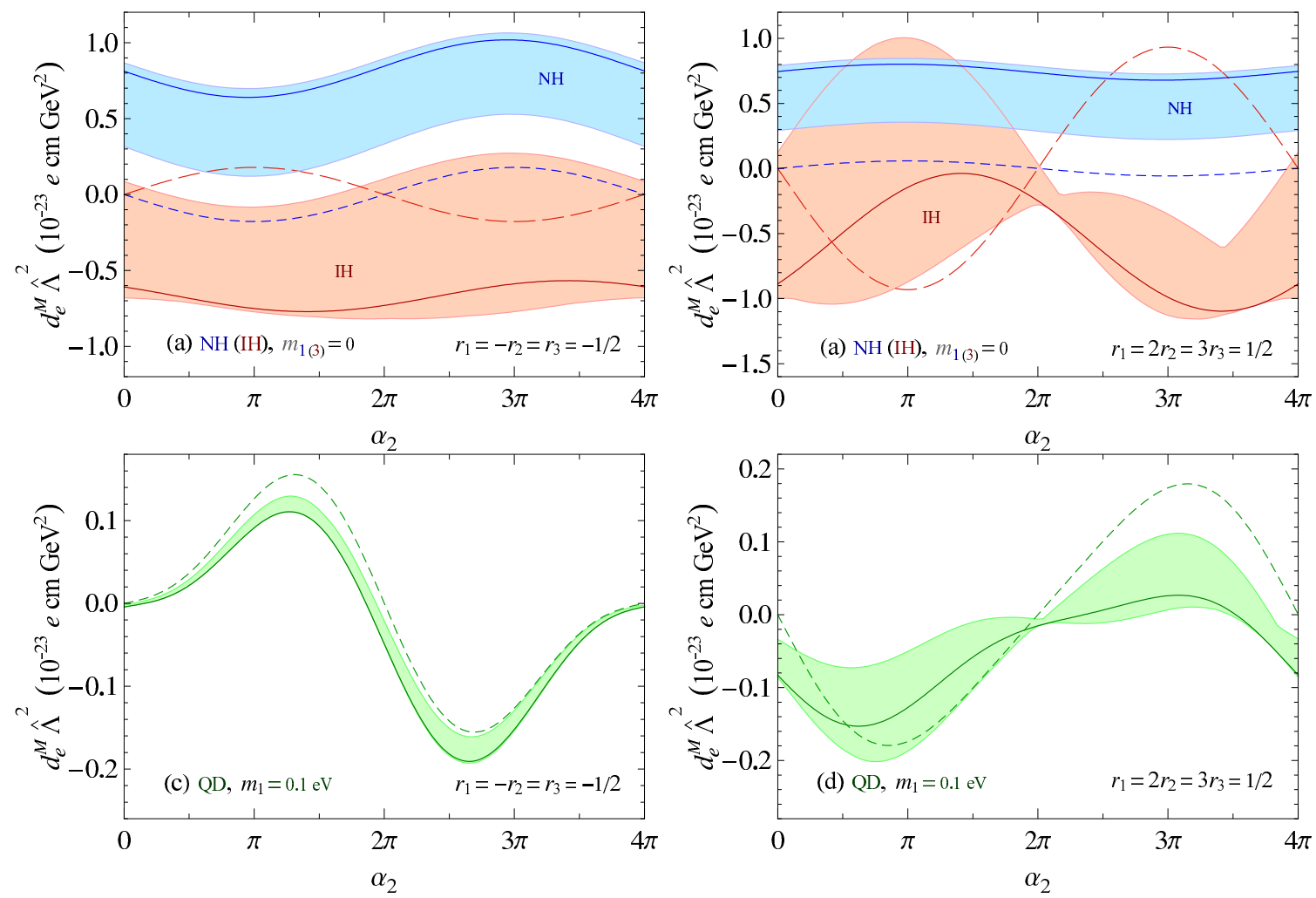

Figure 4. The same as figure 3, except $\nu_{k, R}$ are nondegenerate with $M_{\nu}=\mathcal{M} \operatorname{diag}(1,0.8,1.2)$ and $O=e^{\mathrm{R}}$.

Majorana neutrino $[46,47]$. The information to be gained from the direct neutrino-mass determination in planned tritium $\beta$-decay experiments, with expected sensitivities as low as $0.2 \mathrm{eV}[46,47]$, and the total neutrino mass to be inferred from upcoming cosmological data with improved precision will supply complementary constraints and cross checks.

Before moving on, we would like to make some remarks on the situation in which only two right-handed neutrinos are added into the theory. In that case, $Y_{\nu}$ and $M_{\nu}$ as defined in eq. (2.1) are $3 \times 2$ and $2 \times 2$ matrices, respectively. As a natural consequence [58], it is straightforward to realize from eq. (2.14) that $\left|\operatorname{Det} m_{\nu}\right|=m_{1} m_{2} m_{3}=0$, indicating that one of $m_{1,2,3}$ has to vanish. Another difference is that the $O$ matrix in eq. (2.12) is now $3 \times 2$. Accordingly, with $m_{1}=0$ or $m_{3}=0$ we can write respectively [59]

$$
O=\left(\begin{array}{ll}
0 & 0 \\
1 & 0 \\
0 & 1
\end{array}\right) O_{2} \quad \text { or } \quad O=\left(\begin{array}{ll}
1 & 0 \\
0 & 1 \\
0 & 0
\end{array}\right) O_{2}
$$

where $O_{2}$ is a complex $2 \times 2$ matrix satisfying $O_{2} O_{2}^{\mathrm{T}}=\mathbb{1}_{2}$, where $\mathbb{1}_{2}$ is $2 \times 2$ unit matrix. Thus $\mathrm{O}_{2}$ has 2 free real parameters, whereas $O$ in the presence of 3 right-handed neutrinos has six. All this implies that the specific examples we have provided so far with $m_{1}$ or $m_{3}$ set to zero are applicable to the situation with only 2 right-handed neutrinos, as the 2 parameters of $O_{2}$ are functions of the 6 parameters of $O$ in the case of 3 right-handed 

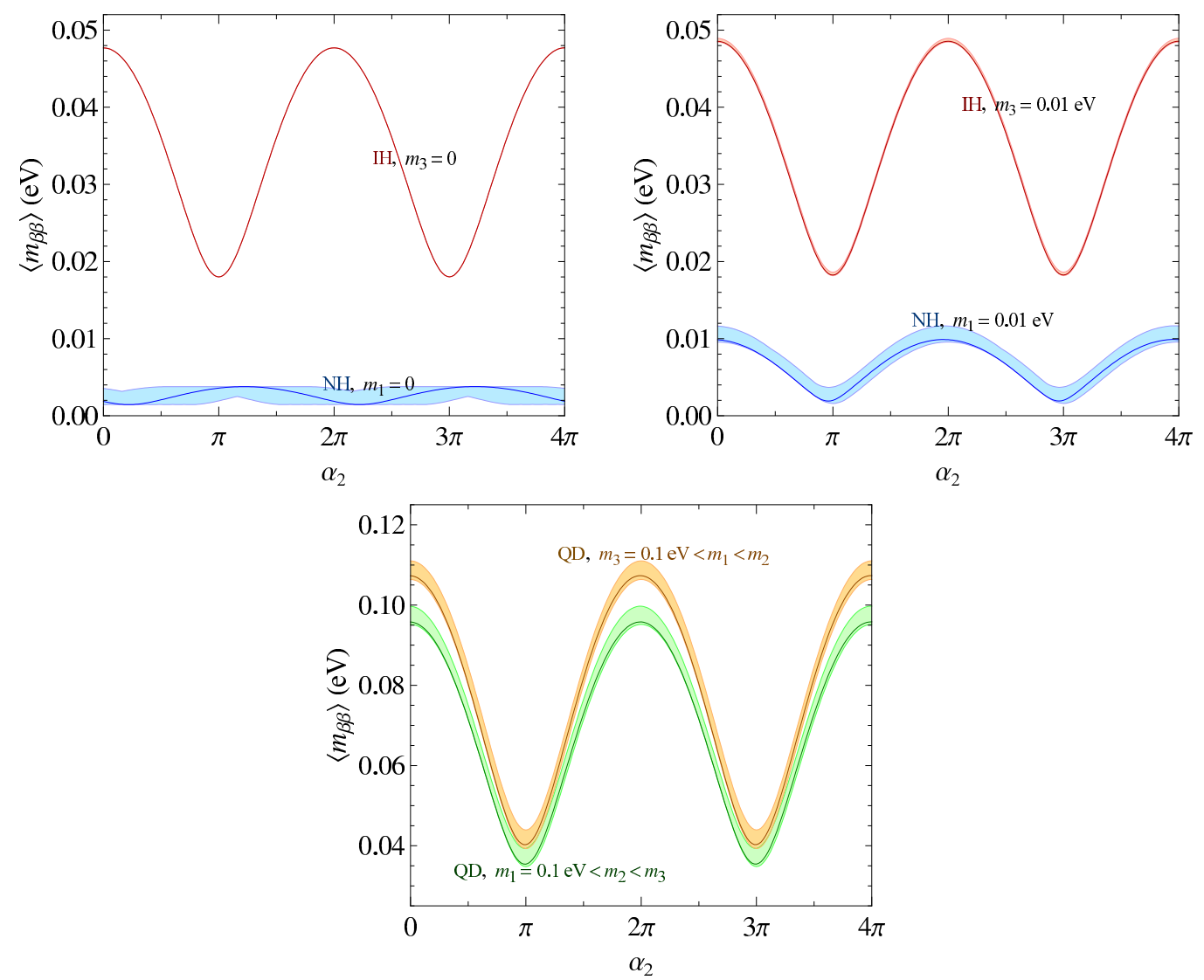

Figure 5. Dependence of effective Majorana mass $\left\langle m_{\beta \beta}\right\rangle$ on $\alpha_{2}$ for $\alpha_{1}=0$, nonzero $\delta$, and some selections of $m_{1}$ or 3 . The bands and solid curves have the same meanings as in previous figures.

neutrinos with $m_{1 \text { or } 3}=0$. We conclude that for $d_{e}$ the situations with 2 and 3 righthanded neutrinos are similar.

\subsection{Muon and tau EDMs}

If neutrinos are of Dirac nature, the muon and tau EDMs will be tiny, like $d_{e}^{\mathrm{D}}$. Therefore here, and in the rest of the section, we suppose that neutrinos are Majorana fermions. Furthermore, for definiteness and simplicity, we consider only the scenario in which the right-handed neutrinos are degenerate, $M_{\nu}=\mathcal{M} \mathbb{1}$, and the orthogonal matrix $O$ is real. For the neutrino parameters, we will adopt the specific values which yielded eq. (4.14) and $\mathcal{M}=6.16(6.22) \times 10^{14} \mathrm{GeV}$ in the $\mathrm{NH}(\mathrm{IH})$ case with $m_{1(3)}=0$.

Accordingly, from eq. (3.10) we easily infer the muon and tau EDMs, respectively, to be

$$
d_{\mu}^{\mathrm{M}}=d_{e}^{\mathrm{M}} \frac{m_{\mu}\left(m_{\tau}^{2}-m_{e}^{2}\right)}{m_{e}\left(m_{\mu}^{2}-m_{\tau}^{2}\right)}, \quad d_{\tau}^{\mathrm{M}}=d_{e}^{\mathrm{M}} \frac{m_{\tau}\left(m_{e}^{2}-m_{\mu}^{2}\right)}{m_{e}\left(m_{\mu}^{2}-m_{\tau}^{2}\right)},
$$

with $d_{e}^{\mathrm{M}}$ in eq. (4.14). Since $d_{\tau}^{\mathrm{M}} \sim-0.06 d_{\mu}^{\mathrm{M}}$ and the experimental information on $d_{\tau}$ is still imprecise [9], we will not deal with it further.

Hence we get $d_{\mu}^{\mathrm{M}}=-2.3(-0.26) \times 10^{-21} \mathrm{GeV}^{2} / \hat{\Lambda}^{2}$. Currently the best measured limit on the muon EDM is $\left|d_{\mu}\right|_{\exp }<1.8 \times 10^{-19} e \mathrm{~cm}$ at $95 \% \mathrm{CL}$, set by the Muon $(g-2)$ 

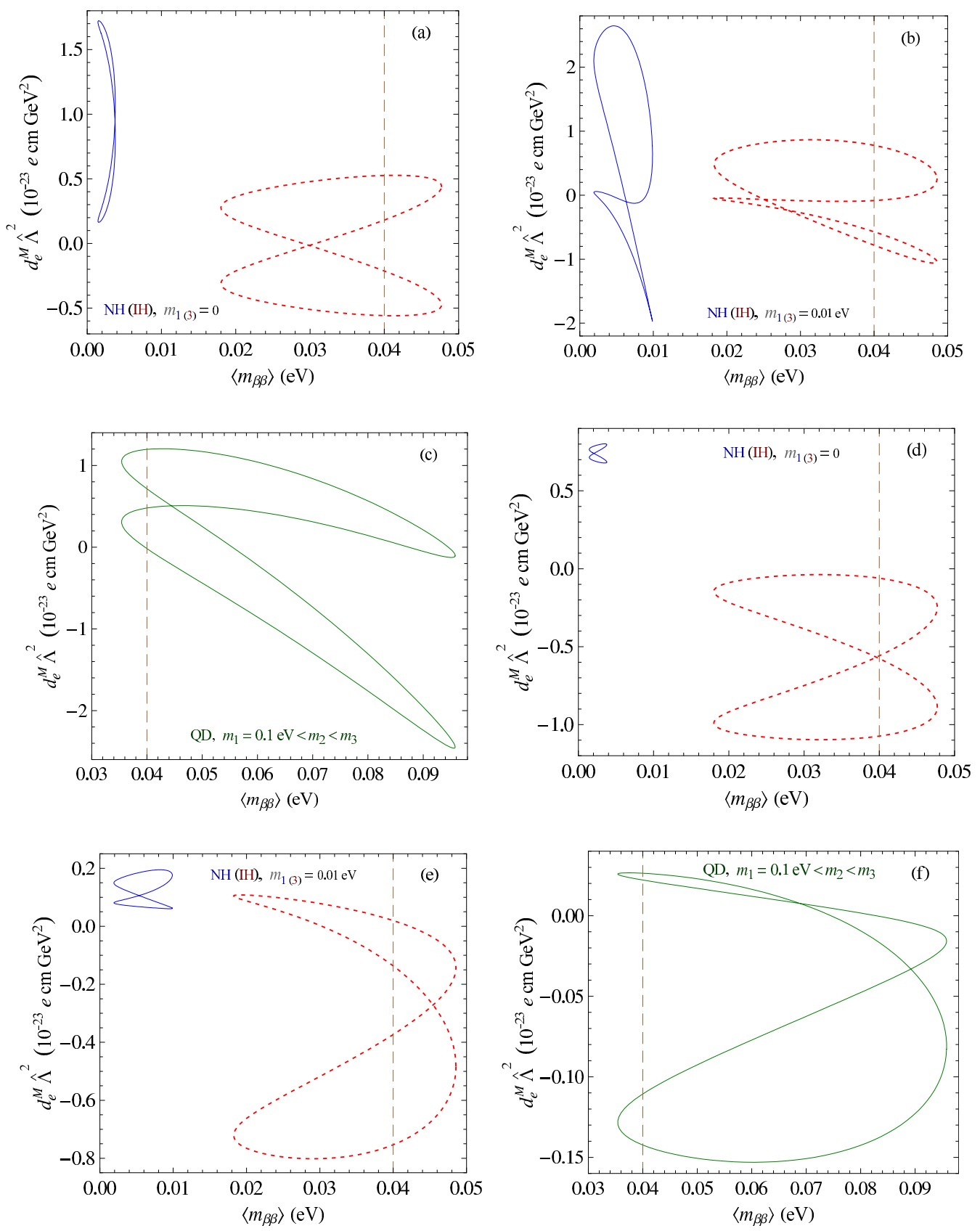

Figure 6. Sample correlations between $d_{e}^{\mathrm{M}} \hat{\Lambda}^{2}$ and $\left\langle m_{\beta \beta}\right\rangle$ over $0 \leq \alpha_{2} \leq 4 \pi$ for $\alpha_{1}=0$ and the central values of $\delta$ in the cases of (a,b,c) degenerate $\nu_{k, R}$ and $O=e^{i \mathrm{R}}$ and (d,e,f) nondegenerate $\nu_{k, R}$ and $O=e^{\mathrm{R}}$, all with $r_{1}=2 r_{2}=3 r_{3}=\frac{1}{2}$, as described in the text. The vertical dashed lines mark a possible sensitivity in future searches for neutrinoless double- $\beta$ within decay the next decade.

Collaboration [60]. This implies

$$
\hat{\Lambda}>0.11(0.038) \mathrm{GeV},
$$

which are not competitive to the bounds in eq. (4.16) from $\left|d_{e}\right|_{\exp }$. 


\section{5 $C P$-violating electron-neutron interactions}

Searches for atomic and molecular EDMs may be sensitive to other mechanisms possibly responsible for them besides the electron EDM, such as the EDMs of nuclei and $C P$-violating electron-nucleon interactions. In this section we are interested in the third possibility, particularly that described by $[5,6]$

$$
\mathcal{L}_{e N}=\frac{-i C_{S} G_{\mathrm{F}}}{\sqrt{2}} \bar{e} \gamma_{5} e \bar{N} N-\frac{i C_{P} G_{\mathrm{F}}}{\sqrt{2}} \bar{e} e \bar{N} \gamma_{5} N-\frac{i C_{T} G_{\mathrm{F}}}{\sqrt{2}} \bar{e} \sigma^{\kappa \omega} \gamma_{5} e \bar{N} \sigma_{\kappa \omega} N .
$$

The recent ACME experiment has set the best limit on the first coupling, $\left|C_{S}\right|_{\exp }<$ $5.9 \times 10^{-9}$ at $90 \% \mathrm{CL}$ [7]. The strictest limits on the other two, $\left|C_{P}\right|_{\exp }<5.1 \times 10^{-7}$ and $\left|C_{T}\right|_{\exp }<1.5 \times 10^{-9}$ at $95 \% \mathrm{CL}$, were based on the latest search for the EDM of the ${ }^{199} \mathrm{Hg}$ atom [61].

These interactions may originate from MFV in the lepton sector as well as the quark sector, which has to be included for a consistent analysis. The Lagrangian for the relevant lowest-order operators is

$$
\begin{aligned}
\mathcal{L}_{\ell q}=\frac{1}{\Lambda^{2}} & \bar{U}_{R} Y_{u}^{\dagger} \bar{\Delta}_{q u 1} i \tau_{2} Q_{L} \bar{E}_{R} Y_{e}^{\dagger} \bar{\Delta}_{\ell 1} L_{L}+\bar{Q}_{L} \bar{\Delta}_{q d 1}^{\dagger} Y_{d} D_{R} \bar{E}_{R} Y_{e}^{\dagger} \bar{\Delta}_{\ell 2} L_{L} \\
& +\bar{U}_{R} \sigma^{\kappa \omega} Y_{u}^{\dagger} \bar{\Delta}_{q u 2} i \tau_{2} Q_{L} \bar{E}_{R} \sigma_{\kappa \omega} Y_{e}^{\dagger} \bar{\Delta}_{\ell 3} L_{L} \\
& \left.+\bar{Q}_{L} \sigma^{\kappa \omega} \bar{\Delta}_{q d 2}^{\dagger} Y_{d} D_{R} \bar{E}_{R} \sigma_{\kappa \omega} Y_{e}^{\dagger} \bar{\Delta}_{\ell 4} L_{L}\right)+ \text { H.c. }
\end{aligned}
$$

where $\bar{\Delta}_{q u \varsigma, q d \varsigma}\left(\bar{\Delta}_{\ell 1, \ell 2, \ell 3, \ell 4}\right)$ are the same in form as $\Delta$ in eq. (2.5) and contain the quark (lepton) Yukawa couplings. The leptonic contributions to $C_{S, P, T}$ turn out to be dominant.

To determine $C_{S}$, we need the matrix elements $\left\langle N\left|m_{q} \bar{q} q\right| N\right\rangle=g_{q}^{N} \bar{u}_{N} u_{N} v$. Thus, we derive

$$
\begin{aligned}
C_{S}= & \frac{16 \sqrt{2} m_{e} \mathcal{M}^{3}}{\Lambda^{2} G_{\mathrm{F}} v^{9}}\left(m_{\tau}^{2}-m_{\mu}^{2}\right)\left(m_{1}-m_{2}\right)\left(m_{2}-m_{3}\right)\left(m_{3}-m_{1}\right) \\
& \times\left[\left(g_{u}^{N}+g_{c}^{N}+\kappa_{u 1} g_{t}^{N}\right) \bar{\xi}_{12}^{\ell 1}-\left(g_{d}^{N}+g_{s}^{N}+\kappa_{d 1} g_{b}^{N}\right) \bar{\xi}_{12}^{\ell 2}\right] J_{\ell},
\end{aligned}
$$

where $\bar{\xi}_{12}^{\ell 1, \ell 2}$ belong to $\bar{\Delta}_{\ell 1, \ell 2}$ and have absorbed the first coefficients $\bar{\xi}_{1}^{u 1, d 1}$ of $\bar{\Delta}_{q u 1, q d 1}$, respectively, and $\kappa_{x} \simeq 1+\left(\bar{\xi}_{2}^{x}+\bar{\xi}_{4}^{x}\right) / \bar{\xi}_{1}^{x}$ are numbers expected to be at most of $\mathcal{O}(1)$. Numerically, we adopt the chiral Lagrangian estimate $[62,63]$

$$
\begin{aligned}
g_{u}^{N} & =0.04(0.12) \times 10^{-3}, & g_{d}^{N} & =0.08(0.21) \times 10^{-3}, \\
g_{s}^{N} & =0.25(2.88) \times 10^{-3}, & g_{c, b, t}^{N} & =0.26(0.05) \times 10^{-3},
\end{aligned}
$$

corresponding to the so-called pion-nucleon sigma term $\sigma_{\pi N}=30(80) \mathrm{MeV}$, which is not yet well-determined [64-67]. ${ }^{4}$ Then, using the maxima of $g_{q}^{N}$ and assuming $\kappa_{x}=1$, we can neglect the $\bar{\xi}_{12}^{\ell 1}$ part in eq. (4.27) to obtain from $\left|C_{S}\right|_{\exp }<5.9 \times 10^{-9}$

$$
\frac{\Lambda}{\left|\bar{\xi}_{12}^{\ell 2}\right|^{1 / 2}}>0.27(0.091) \mathrm{GeV}
$$

\footnotetext{
${ }^{4}$ Lattice QCD computations [64, 65] tend to produce results smaller than those of chiral Lagrangian calculations and some other methods [66, 67]. As a consequence, employing the lattice values of $g_{q}^{N}$ in eq. (4.27) would yield even looser limits than in eq. (4.30).
} 
in the NH (IH) neutrino parameter values specified in the preceding subsection. These restraints are far weaker than those from $\left|d_{e}\right|_{\text {exp }}$.

For $C_{P}$, the expression is the same as that for $C_{S}$ in eq. (4.27), except $g_{q}^{N}$ is replaced by $\varsigma_{q} h_{q}^{N} m_{N} / v$ with $\varsigma_{q}=+1(-1)$ if $q=u, c, t(d, s, b)$ and $h_{q}^{N}$ defined by $\left\langle N\left|m_{q} \bar{q} \gamma_{5} q\right| N\right\rangle=h_{q}^{N} m_{N} \bar{u}_{N} \gamma_{5} u_{N}$. Since for mercury $C_{P}$ is estimated to be mostly from the neutron contribution [6], we focus on it. Ignoring the effects of $h_{c, b, t}^{n}$, we can relate $h_{u, d, s}^{n}$ to the axial-vector charges $g_{A}^{(0,3,8)}$ by $6 h_{u}^{n}=2 g_{A}^{(0)}-3 g_{A}^{(3)}+g_{A}^{(8)}, 6 h_{d}^{n}=2 g_{A}^{(0)}+3 g_{A}^{(3)}+g_{A}^{(8)}$, and $3 h_{s}^{n}=g_{A}^{(0)}-g_{A}^{(8)}$, where $g_{A}^{(0)}=0.33 \pm 0.06, g_{A}^{(3)}=1.270 \pm 0.003$, and $g_{A}^{(8)}=0.58 \pm 0.03$ were measured in baryon $\beta$-decay and deep inelastic scattering experiments [68]. Taking $\bar{\xi}_{12}^{\ell 1}=\bar{\xi}_{12}^{\ell 2}$ and maximizing $C_{P}$, we obtain from $\left|C_{P}\right|_{\exp }<5.1 \times 10^{-7}$

$$
\frac{\Lambda}{\left|\bar{\xi}_{12}^{\ell 2}\right|^{1 / 2}}>0.020(0.0068) \mathrm{GeV}
$$

less restrictive than eq. (4.30) by more than an order of magnitude.

To evaluate $C_{T}$, we need the matrix elements $\left\langle N\left|\bar{q} \sigma^{\kappa \omega} q\right| N\right\rangle=\rho_{N}^{q} \bar{u}_{N} \sigma^{\kappa \omega} u_{N}$, where $\rho_{N}^{q}$ have the values in eq. (4.3) for light quarks, assuming isospin symmetry, and vanish for heavier quarks. This leads us to

$$
C_{T}=\frac{32 \sqrt{2} m_{e} \mathcal{M}^{3}}{\Lambda^{2} G_{\mathrm{F}} v^{10}}\left(m_{\tau}^{2}-m_{\mu}^{2}\right)\left(m_{1}-m_{2}\right)\left(m_{2}-m_{3}\right)\left(m_{3}-m_{1}\right) \rho_{u}^{n} m_{u} \bar{\xi}_{12}^{\ell 3} J_{\ell}
$$

where $\bar{\xi}_{12}^{\ell 3}$ belongs to $\bar{\Delta}_{\ell 3}$ and has absorbed $\bar{\xi}_{1}^{u 2}$ from $\bar{\Delta}_{q u 2}$. The contributions of the downtype quarks cancel due to the relation $\bar{q} \sigma^{\kappa \omega} \gamma_{5} q \bar{e} \sigma_{\kappa \omega} e=\bar{q} \sigma^{\kappa \omega} q \bar{e} \sigma_{\kappa \omega} \gamma_{5} e$. Hence, with the largest $m_{u}$ from section 4.1 and $\rho_{n}^{u}=-0.78$, we get from $\left|C_{T}\right|_{\exp }<1.5 \times 10^{-9}$

$$
\frac{\Lambda}{\left|\bar{\xi}_{12}^{\ell 4}\right|^{1 / 2}}>0.033(0.011) \mathrm{GeV} \text {, }
$$

comparable to eq. (4.31).

\subsection{Muon $g-2, \mu \rightarrow e \gamma$, nuclear $\mu \rightarrow e$ conversion, $\bar{B} \rightarrow X_{s} \gamma$}

The MFV coefficient $\xi_{12}^{\ell}$ that determines the electron EDM also enters the anomalous magnetic moment of the muon $\left(g_{\mu}-2\right)$ and the rates of the radiative decay $\mu \rightarrow e \gamma$ and nuclear $\mu \rightarrow e$ conversion, the latter two being still unobserved. Since $g_{\mu}-2$ has been very precisely measured and the experimental limits of the flavor-changing transitions are stringent, it is important to check if these processes can yield stronger bounds on $\hat{\Lambda}=\Lambda /\left|\xi_{12}^{\ell}\right|^{1 / 2}$ than those evaluated in the preceding subsections. Although the other $\xi_{r \neq 12,16}^{\ell}$ terms may contribute to these processes as well and therefore may reduce the impact of the $\xi_{12}^{\ell}$ term, one also cannot rule out the possibility of a scenario in which the latter dominates the other contributions.

The anomalous magnetic moment $a_{l}$ of lepton $l$ is described by $\mathcal{L}_{a_{l}}=$ $\left[e a_{l} /\left(4 m_{l}\right)\right] \bar{l} \sigma^{\kappa \omega} l F_{\kappa \omega}$. From eq. (3.3) we have

$$
\mathcal{L}_{E_{i} \rightarrow E_{k} \gamma}=\frac{e}{2 \Lambda^{2}} \bar{E}_{k} \sigma_{\kappa \omega}\left\{m_{E_{k}}\left(\Delta_{\ell}\right)_{k i}+m_{E_{i}}\left(\Delta_{\ell}\right)_{i k}^{*}-\left[m_{E_{k}}\left(\Delta_{\ell}\right)_{k i}-m_{E_{i}}\left(\Delta_{\ell}\right)_{i k}^{*}\right] \gamma_{5}\right\} E_{i} F^{\kappa \omega},
$$


where $\left(E_{1}, E_{2}, E_{3}\right)=(e, \mu, \tau)$ and $\Delta_{\ell}=\Delta_{\ell 1}-\Delta_{\ell 2}$. It follows that

$$
a_{E_{k}}=\frac{4 m_{E_{k}}^{2}}{\Lambda^{2}} \operatorname{Re}\left(\Delta_{\ell}\right)_{k k} .
$$

Thus, with the NH neutrino parameter values specified in section 4.4, we have

$$
a_{\mu}=\frac{4 m_{\mu}^{2}}{\Lambda^{2}} \operatorname{Re}\left(\Delta_{\ell}\right)_{22}=\left(45 \xi_{1}^{\ell}+23 \xi_{2}^{\ell}+20 \xi_{4}^{\ell}+0.00085 \xi_{8}^{\ell}+0.00094 \xi_{12}^{\ell}\right) \frac{\mathrm{GeV}^{2}}{10^{3} \Lambda^{2}}
$$

where terms with numerical factors much smaller than that of $\xi_{12}^{\ell}$ have been dropped. The corresponding numbers in the $\mathrm{IH}$ case are roughly similar. Currently the experimental and SM values differ by $a_{\mu}^{\exp }-a_{\mu}^{\mathrm{SM}}=(249 \pm 87) \times 10^{-11}[69]$, which suggests that we can require $\left|a_{\mu}\right|<3.4 \times 10^{-9}$. For the $\xi_{12}^{\ell}$ term alone, this translates into the rather loose limit $\hat{\Lambda}>17 \mathrm{GeV}$, which may be weakened in the presence of the other terms in eq. (4.36).

From eq. (4.34), one can also calculate the branching ratio $\mathcal{B}(\mu \rightarrow e \gamma)$ of $\mu \rightarrow e \gamma$. In the $m_{e}=0$ limit

$$
\mathcal{B}(\mu \rightarrow e \gamma)=\frac{\tau_{\mu} e^{2} m_{\mu}^{5}}{4 \pi \Lambda^{4}}\left|\left(\Delta_{\ell}\right)_{21}\right|^{2},
$$

where $\tau_{\mu}$ is the muon lifetime. In the $\mathrm{NH}$ case

$$
\left(\Delta_{\ell}\right)_{21}=(0.061-0.1 i) \xi_{2}^{\ell}+(0.011-0.11 i) \xi_{4}^{\ell}-\left[(26+48 i) \xi_{8}^{\ell}+(6+51 i) \xi_{12}^{\ell}\right] \times 10^{-7},
$$

where again terms with numerical factors less than that of $\xi_{12}^{\ell}$ have been ignored. The $\left(\Delta_{\ell}\right)_{21}$ numbers in the IH case are comparable in size. If only $\xi_{12}^{\ell}$ is nonvanishing in $\left(\Delta_{\ell}\right)_{21}$, then the experimental bound $\mathcal{B}(\mu \rightarrow e \gamma)_{\exp }<5.7 \times 10^{-13}[70]$ implies

$$
\hat{\Lambda}>2.0 \mathrm{TeV} \text {. }
$$

This is stronger by up to $\sim 20$ times than those in eq. (4.16) from the electron EDM data. However, the other terms in $\left(\Delta_{\ell}\right)_{21}$, some of which are potentially much bigger than the $\xi_{12}^{\ell}$ contribution, can in principle decrease the impact of the latter, thereby lessening the restriction on $\hat{\Lambda}$. Consequently, $d_{e}$ provides a less ambiguous probe for $\hat{\Lambda}$.

Measurements on $\mu \rightarrow e$ conversion in nuclei can provide constraints on new physics competitive to those from $\mu \rightarrow e \gamma$ searches [71]. The relation between the rates of $\mu \rightarrow e$ conversion and $\mu \rightarrow e \gamma$ produced by possible new physics is available from ref. [72]. Assuming that the MFV dipole interactions described by eq. (3.2) saturate $\mu \rightarrow e$ conversion in nucleus $\mathcal{N}$, we can express its rate divided by the rate $\omega_{\text {capt }}^{\mathcal{N}}$ of $\mu$ capture in $\mathcal{N}$ as

$$
\mathcal{B}(\mu \mathcal{N} \rightarrow e \mathcal{N})=\frac{e^{2} m_{\mu}^{5}\left|\left(\Delta_{\ell}\right)_{21} D_{\mathcal{N}}\right|^{2}}{4 \Lambda^{4} \omega_{\text {capt }}^{\mathcal{N}}},
$$

where $D_{\mathcal{N}}$ represents the dimensionless overlap integral for $\mathcal{N}$ and for the $\mathrm{NH}$ parameter choices $\left(\Delta_{\ell}\right)_{21}$ is given in eq. (4.38). Based on the existing experimental limits on $\mu \rightarrow e$ transition in various nuclei [9] and the corresponding $D_{\mathcal{N}}$ and $\omega_{\text {capt }}^{\mathcal{N}}$ values [72], significant restrictions can be expected from $\mathcal{B}(\mu \mathrm{Ti} \rightarrow e \mathrm{Ti})_{\exp }<6.1 \times 10^{-13}[73]$ and $\mathcal{B}(\mu \mathrm{Au} \rightarrow$ 
$e \mathrm{Au})_{\exp }<7 \times 10^{-13}[9]$. From these data, if only the $\xi_{12}^{\ell}$ term in $\left(\Delta_{\ell}\right)_{21}$ is nonvanishing, employing $D_{\mathrm{Ti}}=0.087, D_{\mathrm{Au}}=0.189, \omega_{\text {capt }}^{\mathrm{Ti}}=2.59 \times 10^{6} / \mathrm{s}$, and $\omega_{\text {capt }}^{\mathrm{Au}}=13.07 \times 10^{6} / \mathrm{s}[72]$, we extract

$$
\hat{\Lambda}_{\mathrm{Ti}}>0.49 \mathrm{TeV}, \quad \hat{\Lambda}_{\mathrm{Au}}>0.47 \mathrm{TeV},
$$

which are stricter than the results in eq. (4.30) by up to a few times, but weaker than eq. (4.39). Upcoming searches for $\mu \rightarrow e$ in the next several years will, if it still eludes detection, lower the limits to the $10^{-16}$ level or better [71], which will push $\hat{\Lambda}$ higher. Nevertheless, since again the other $\xi_{r}^{\ell}$ terms are generally present in $\left(\Delta_{\ell}\right)_{21}$, these bounds on $\hat{\Lambda}$ are not unambiguous. Thus $d_{e}$ provides the best probe for $\hat{\Lambda}$ in connection with $C P$ violation.

Since there is a possibility that the MFV scales in the lepton and quark sectors are equal or related to each other, it is of interest to check if there are any quark processes that can also offer bounds stronger than those on $\hat{\Lambda}$ from $d_{e}$. Since, as we saw in section 4.1, the neutron EDM could not provide a competitive constraint, we need to look at other processes. The most stringent restriction on the quark MFV scale turns out to be from the rare decay $\bar{B} \rightarrow X_{s} \gamma$ [21]. Its experimental and SM branching ratios are $\mathcal{B}(\bar{B} \rightarrow$ $\left.X_{s} \gamma\right)_{\exp }=(3.43 \pm 0.22) \times 10^{-4}[74,75]$ and $\mathcal{B}\left(\bar{B} \rightarrow X_{s} \gamma\right)_{\mathrm{SM}}=(3.15 \pm 0.23) \times 10^{-4}[76]$ both for the photon energy $E_{\gamma}>1.6 \mathrm{GeV}$. To isolate the MFV contribution, we adopt from ref. [21] the relation

$$
\mathcal{B}\left(\bar{B} \rightarrow X_{s} \gamma\right)_{\exp } \simeq\left(1-2.4 C_{7 \gamma}^{\mathrm{MFV}}\right) \mathcal{B}\left(\bar{B} \rightarrow X_{s} \gamma\right)_{\mathrm{SM}}
$$

where $C_{7 \gamma}^{\mathrm{MFV}}$ is evaluated at $\mu=m_{W}$ and enters the effective Lagrangian

$$
\mathcal{L}_{b \rightarrow s \gamma}=\frac{e G_{\mathrm{F}} m_{b}}{8 \sqrt{2} \pi^{2}} V_{t s}^{*} V_{t b}\left(C_{7 \gamma}^{\mathrm{SM}}+C_{7 \gamma}^{\mathrm{MFV}}\right) \bar{s} \sigma^{\kappa \omega}\left(1+\gamma_{5}\right) b F_{\kappa \omega},
$$

implying that

$$
C_{7 \gamma}^{\mathrm{MFV}}=\frac{4 \sqrt{2} \pi^{2}}{\Lambda^{2} G_{\mathrm{F}}} \frac{\left(\Delta_{q d}\right)_{32}^{*}}{V_{t s}^{*} V_{t b}}
$$

For the central values of the quark masses quoted in section 4.1

$$
\frac{\left(\Delta_{q d}\right)_{32}^{*}}{V_{t s}^{*} V_{t b}}=\xi_{2}^{d} y_{t}^{2}+\xi_{4}^{d} y_{t}^{4}+y_{b}^{2}\left(\xi_{7}^{d} y_{t}^{2}+\xi_{8}^{d} y_{t}^{4}+\xi_{9}^{d} y_{t}^{4}+\xi_{12}^{d} y_{t}^{6}\right)
$$

where the imaginary parts and other $\xi_{r}^{d}$ terms are negligible, $y_{t}^{2} \simeq 1$, and $y_{b}^{2} \simeq 0.0003$. Combining the errors in quadrature for the ratio of branching ratios in eq. (4.42) and assuming that $\xi_{r \neq 12}^{d}=0$, we obtain at $90 \% \mathrm{CL}$

$$
\frac{\Lambda}{\left|\xi_{12}^{d}\right|^{1 / 2}}>0.19(0.11) \mathrm{TeV}
$$

if $C_{7 \gamma}^{\mathrm{MFv}}$ has destructive (constructive) interference with the SM term. These numbers are somewhat lower than those in eq. (4.16) and, as in the lepton cases, may go down in the presence of the other $\xi_{r}^{d}$ terms in eq. (4.45). 


\section{Conclusions}

We have explored $C P$ violation beyond the SM via fermion EDMs under the framework of minimal flavor violation. The new physics scenarios covered are the standard model slightly expanded with the addition of three right-handed neutrinos and its extension including the seesaw mechanism for endowing neutrinos with light mass. Addressing the quark sector first, we find that the present empirical limit on the neutron EDM implies only a loose constraint on the scale of quark MFV. Moreover, we show that the impact of MFV on the contribution of the strong theta-term to the neutron EDM is insignificant. Turning to the lepton sector, we demonstrate that the current EDM data also yield unimportant restraints on the leptonic MFV scale if neutrinos are of Dirac nature. In contrast, if neutrinos are Majorana particles, the constraints become tremendously more stringent and, in light of the latest search for $d_{e}$ by ACME, restrict the MFV scale to above a few hundred GeV or more. Furthermore, $d_{e}$ can be connected in a complementary way to neutrinoless double- $\beta$ decay if it is induced mainly or solely by the exchange of a light Majorana neutrino. We find in addition that constraints on the MFV scale inferred from the $C P$-violating electronnucleon couplings probed by ACME and the most recent search for the EDM of mercury are relatively weak as well. Finally, we take into account potential restrictions from the measurements on the muon $g-2$, radiative decays $\mu \rightarrow e \gamma$ and $\bar{B} \rightarrow X_{s} \gamma$, and $\mu \rightarrow e$ conversion in nuclei, which are not sensitive to $C P$ violation.

\section{Acknowledgments}

This research was supported in part by the MOE Academic Excellence Program (Grant No. 102R891505) and NSC of ROC and by NNSF (Grant No. 11175115) and Shanghai Science and Technology Commission (Grant No. 11DZ2260700) of PRC.

\section{A Evaluation of some products of A and B matrices}

From the Cayley-Hamilton identity in eq. (2.4) with $X=a \mathrm{~A}+b \mathrm{~B}$, where and $a$ and $b$ are free parameters, one can extract [34]

$$
\begin{aligned}
A^{2} B+A B A+B A^{2}= & A^{2}\langle B\rangle+(A B+B A)\langle A\rangle+A(\langle A B\rangle-\langle A\rangle\langle B\rangle)+\frac{1}{2}\left(\left\langle A^{2}\right\rangle-\langle A\rangle^{2}\right) B \\
& +\mathbb{1}\left[\frac{1}{2}\left(\langle A\rangle^{2}-\left\langle A^{2}\right\rangle\right)\langle B\rangle+\left\langle A^{2} B\right\rangle-\langle A\rangle\langle A B]\right.
\end{aligned}
$$

and an analogous expression for $\mathrm{ABB}+\mathrm{BAB}+\mathrm{BBA}$, where $\langle\cdots\rangle=\operatorname{Tr}(\cdots)$. These relations can be used to derive other combinations of $A$ and $B$. For instance, by replacing $B$ with $B^{2}$ $([B, A B])$ in eq. (A.1), we can write $A^{2} B^{2}+A B^{2} A+B^{2} A^{2} \quad\left(A^{2} B A B+A B A B A+B A B A^{2}-\right.$ $\left.A^{2} B^{2} A-A B^{2} A^{2}-A^{3} B^{2}\right)$ in terms of lower-ordered products of these matrices. After further algebra, we arrive at

$$
\begin{aligned}
\mathrm{A}^{2} \mathrm{BAB}^{2}= & \zeta_{1} \mathbb{1}+\zeta_{2} \mathrm{~A}+\zeta_{3} \mathrm{~B}+\zeta_{4} \mathrm{~A}^{2}+\zeta_{5} \mathrm{~B}^{2}+\zeta_{6} \mathrm{AB}+\zeta_{7} \mathrm{BA}+\zeta_{8} \mathrm{ABA}+\zeta_{9} \mathrm{BA}^{2} \\
& +\zeta_{10} \mathrm{BAB}+\zeta_{11} \mathrm{AB}^{2}+\zeta_{12} \mathrm{ABA}^{2}+\zeta_{13} \mathrm{~A}^{2} \mathrm{~B}^{2}+\zeta_{14} \mathrm{~B}^{2} \mathrm{~A}^{2}+\zeta_{15} \mathrm{~B}^{2} \mathrm{AB} \\
& +\zeta_{16} \mathrm{AB}^{2} \mathrm{~A}^{2}+\zeta_{17} \mathrm{~B}^{2} \mathrm{~A}^{2} \mathrm{~B}
\end{aligned}
$$


where

$$
\begin{aligned}
& \zeta_{1}=\frac{\left\langle A^{2} B A B^{2}\right\rangle+\langle A B\rangle\left\langle A^{2} B^{2}\right\rangle+\left\langle A^{2} B\right\rangle\left\langle A B^{2}\right\rangle}{3}+\langle A\rangle\langle B\rangle \frac{4\left\langle A^{2}\right\rangle\left\langle B^{2}\right\rangle-6\left\langle A^{2} B^{2}\right\rangle-3\langle A\rangle^{2}\langle B\rangle^{2}}{6} \\
& +\frac{\left(\langle B\rangle^{3}-\langle B\rangle\left\langle B^{2}\right\rangle\right) \operatorname{Det} A+\left(\langle A\rangle^{3}-\langle A\rangle\left\langle A^{2}\right\rangle\right) \operatorname{Det} B}{6}+\langle A B\rangle \frac{13\langle A\rangle^{2}\langle B\rangle^{2}-3\left\langle A^{2}\right\rangle\left\langle B^{2}\right\rangle}{12} \\
& +\langle A B\rangle \frac{5\langle A\rangle^{2}\left\langle B^{2}\right\rangle+\langle B\rangle^{2}\left\langle A^{2}\right\rangle-8\langle A\rangle\left\langle A B^{2}\right\rangle-4\langle B\rangle\left\langle A^{2} B\right\rangle}{12}+\left\langle A B^{2}\right\rangle\langle B\rangle \frac{\langle A\rangle^{2}-\left\langle A^{2}\right\rangle}{6} \\
& -\langle A\rangle\left\langle B^{2}\right\rangle \frac{\langle A\rangle^{2}\langle B\rangle+2\left\langle A^{2} B\right\rangle}{6}, \\
& \zeta_{2}=\frac{-\left\langle A^{2}\right\rangle \operatorname{Det} B}{3}+\langle A B\rangle \frac{4\left\langle A B^{2}\right\rangle-5\langle A\rangle\langle B\rangle^{2}-3\langle A\rangle\left\langle B^{2}\right\rangle}{6}+\langle B\rangle\left\langle B^{2}\right\rangle \frac{7\langle A\rangle^{2}+\left\langle A^{2}\right\rangle}{12} \\
& -\langle B\rangle \frac{2\langle A\rangle\left\langle A B^{2}\right\rangle+\left\langle A^{2} B^{2}\right\rangle}{3}+\frac{\langle B\rangle^{2}\left\langle A^{2} B\right\rangle}{3}+\langle B\rangle^{3} \frac{9\langle A\rangle^{2}-\left\langle A^{2}\right\rangle}{12}, \\
& \zeta_{3}=\frac{-\left\langle B^{2}\right\rangle \text { DetA }}{3}+\langle A B\rangle \frac{2\left\langle A^{2} B\right\rangle-5\langle A\rangle^{2}\langle B\rangle-\left\langle A^{2}\right\rangle\langle B\rangle}{6}+\langle A\rangle\left\langle A^{2}\right\rangle \frac{3\langle B\rangle^{2}-\left\langle B^{2}\right\rangle}{12} \\
& -\langle A\rangle \frac{\left\langle A^{2} B\right\rangle\langle B\rangle+\left\langle A^{2} B^{2}\right\rangle}{3}+\left\langle A B^{2}\right\rangle \frac{\langle A\rangle^{2}+\left\langle A^{2}\right\rangle}{6}+\langle A\rangle^{3} \frac{9\langle B\rangle^{2}+\left\langle B^{2}\right\rangle}{12}, \\
& \zeta_{4}=\frac{\langle A\rangle \operatorname{DetB}}{3}+\left\langle B^{2}\right\rangle \frac{2\langle A B\rangle-7\langle A\rangle\langle B\rangle}{6}-\frac{\langle A\rangle\langle B\rangle^{3}}{6}+\frac{\left\langle A B^{2}\right\rangle\langle B\rangle}{3}, \\
& \zeta_{5}=\frac{\langle\mathrm{B}\rangle \operatorname{Det} \mathrm{A}}{3}+\left\langle\mathrm{A}^{2}\right\rangle \frac{2\langle\mathrm{AB}\rangle-5\langle\mathrm{~A}\rangle\langle\mathrm{B}\rangle}{6}-\frac{\langle\mathrm{A}\rangle^{3}\langle\mathrm{~B}\rangle}{6}, \\
& \zeta_{6}=\left\langle A^{2}\right\rangle \frac{\left\langle B^{2}\right\rangle+\langle B\rangle^{2}}{6}-\langle A\rangle^{2} \frac{\left\langle B^{2}\right\rangle+7\langle B\rangle^{2}}{6}+\frac{2\langle A\rangle\left\langle A B^{2}\right\rangle+\langle B\rangle\left\langle A^{2} B\right\rangle-2\left\langle A^{2} B^{2}\right\rangle}{3}, \\
& \zeta_{7}=\left\langle A^{2}\right\rangle \frac{\left\langle B^{2}\right\rangle-\langle B\rangle^{2}}{12}-\langle A\rangle^{2} \frac{5\left\langle B^{2}\right\rangle+11\langle B\rangle^{2}}{12}+\frac{2\langle A\rangle\left\langle A B^{2}\right\rangle+\langle B\rangle\left\langle A^{2} B\right\rangle-\left\langle A^{2} B^{2}\right\rangle}{3}, \\
& \zeta_{8}=\langle A\rangle \frac{5\langle B\rangle^{2}+3\left\langle B^{2}\right\rangle}{6}-\frac{2\left\langle A B^{2}\right\rangle}{3}, \quad \zeta_{9}=\frac{\langle A\rangle\left\langle B^{2}\right\rangle-\left\langle A B^{2}\right\rangle}{3} \\
& \zeta_{10}=\langle B\rangle \frac{5\langle A\rangle^{2}+\left\langle A^{2}\right\rangle}{6}-\frac{\left\langle A^{2} B\right\rangle}{3}, \quad \zeta_{11}=\frac{\left\langle A^{2} B\right\rangle-\left\langle A^{2}\right\rangle\langle B\rangle}{3}, \\
& \zeta_{12}=\frac{-\langle\mathrm{B}\rangle^{2}}{2}-\frac{\left\langle\mathrm{B}^{2}\right\rangle}{6}, \quad \zeta_{13}=\frac{4\langle\mathrm{~A}\rangle\langle\mathrm{B}\rangle+\langle\mathrm{AB}\rangle}{3}, \\
& \zeta_{14}=\langle\mathrm{A}\rangle\langle\mathrm{B}\rangle-\frac{\langle\mathrm{AB}\rangle}{3}, \quad \zeta_{15}=\frac{-\langle\mathrm{A}\rangle^{2}}{2}-\frac{\left\langle\mathrm{A}^{2}\right\rangle}{6}, \\
& \zeta_{16}=\frac{2\langle\mathrm{~B}\rangle}{3}, \quad \zeta_{17}=\frac{2\langle\mathrm{~A}\rangle}{3} .
\end{aligned}
$$

The Hermiticity of $\mathrm{A}$ and $\mathrm{B}$ implies that all the traces and determinants in $\zeta_{1,2, \cdots, 17}$ are purely real, except $\left\langle A^{2} B A B^{2}\right\rangle$ in $\zeta_{1}$ which has an imaginary component

$$
J_{\xi}=\operatorname{Im}\left\langle\mathrm{A}^{2} \mathrm{BAB}^{2}\right\rangle=\frac{i}{2} \operatorname{Det}[\mathrm{A}, \mathrm{B}]
$$


obtainable from the Cayley-Hamilton identity

$$
[A, B]^{3}=\mathbb{1} \operatorname{Det}[A, B]+\frac{1}{2}[A, B]\left(\left\langle[A, B]^{2}\right\rangle-\langle[A, B]\rangle^{2}\right)+[A, B]^{2}\langle[A, B]\rangle .
$$

Clearly the reduction of $\mathrm{A}^{2} \mathrm{BA} \mathrm{B}^{2}$ into a sum of matrix products with lower orders causes the coefficient $\zeta_{1}$ to gain an imaginary component equal to $J_{\xi}$. It follows that higher-order matrix products containing $\mathrm{A}^{2} \mathrm{BAB}^{2}$ will lead to contributions to the coefficients $\xi_{r}$ with imaginary parts which are always proportional to $J_{\xi}$

Open Access. This article is distributed under the terms of the Creative Commons Attribution License (CC-BY 4.0), which permits any use, distribution and reproduction in any medium, provided the original author(s) and source are credited.

\section{References}

[1] X.-G. He, B.H.J. McKellar and S. Pakvasa, The Neutron Electric Dipole Moment, Int. J. Mod. Phys. A 4 (1989) 5011 [Erratum ibid. A 6 (1991) 1063] [inSPIRE].

[2] W. Bernreuther and M. Suzuki, The electric dipole moment of the electron, Rev. Mod. Phys. 63 (1991) 313 [Erratum ibid. 64 (1992) 633] [InSPIRE].

[3] M. Pospelov and A. Ritz, Electric dipole moments as probes of new physics, Annals Phys. 318 (2005) 119 [hep-ph/0504231] [INSPIRE].

[4] T. Fukuyama, Searching for New Physics beyond the Standard Model in Electric Dipole Moment, Int. J. Mod. Phys. A 27 (2012) 1230015 [arXiv:1201.4252] [INSPIRE].

[5] J. Engel, M.J. Ramsey-Musolf and U. van Kolck, Electric Dipole Moments of Nucleons, Nuclei and Atoms: The Standard Model and Beyond, Prog. Part. Nucl. Phys. 71 (2013) 21 [arXiv: 1303.2371] [INSPIRE].

[6] J.S.M. Ginges and V.V. Flambaum, Violations of fundamental symmetries in atoms and tests of unification theories of elementary particles, Phys. Rept. 397 (2004) 63 [physics/0309054] [INSPIRE].

[7] ACME collaboration, J. Baron et al., Order of Magnitude Smaller Limit on the Electric Dipole Moment of the Electron, Science 343 (2014) 269 [arXiv:1310.7534] [InSPIRE].

[8] M. Pospelov and A. Ritz, CKM benchmarks for electron EDM experiments, Phys. Rev. D 89 (2014) 056006 [arXiv:1311.5537] [InSPIRE].

[9] Particle Data Group collaboration, J. Beringer et al., Review of Particle Physics (RPP), Phys. Rev. D 86 (2012) 010001 [INSPIRE].

[10] M.B. Gavela, A. Le Yaouanc, L. Oliver, O. Pene, J.C. Raynal et al., CP Violation Induced by Penguin Diagrams and the Neutron Electric Dipole Moment, Phys. Lett. B 109 (1982) 215 [INSPIRE].

[11] I.B. Khriplovich and A.R. Zhitnitsky, What Is the Value of the Neutron Electric Dipole Moment in the Kobayashi-Maskawa Model?, Phys. Lett. B 109 (1982) 490 [INSPIRE].

[12] J.O. Eeg and I. Picek, Two Loop Diagrams for the Electric Dipole Moment of the Neutron, Nucl. Phys. B 244 (1984) 77 [InSPIRE].

[13] B.H.J. McKellar, S.R. Choudhury, X.-G. He and S. Pakvasa, The Neutron Electric Dipole Moment in the Standard $K^{-} m$ Model, Phys. Lett. B 197 (1987) 556 [INSPIRE]. 
[14] R.S. Chivukula and H. Georgi, Composite Technicolor Standard Model, Phys. Lett. B 188 (1987) 99 [INSPIRE].

[15] L.J. Hall and L. Randall, Weak scale effective supersymmetry, Phys. Rev. Lett. 65 (1990) 2939 [INSPIRE].

[16] A.J. Buras, P. Gambino, M. Gorbahn, S. Jager and L. Silvestrini, Universal unitarity triangle and physics beyond the standard model, Phys. Lett. B 500 (2001) 161 [hep-ph/0007085] [INSPIRE].

[17] A.J. Buras, Minimal flavor violation, Acta Phys. Polon. B 34 (2003) 5615 [hep-ph/0310208] [INSPIRE].

[18] S. Davidson and F. Palorini, Various definitions of Minimal Flavour Violation for Leptons, Phys. Lett. B 642 (2006) 72 [hep-ph/0607329] [INSPIRE].

[19] A.L. Kagan, G. Perez, T. Volansky and J. Zupan, General Minimal Flavor Violation, Phys. Rev. D 80 (2009) 076002 [arXiv:0903.1794] [inSPIRE].

[20] A.J. Buras and J. Girrbach, Towards the Identification of New Physics through Quark Flavour Violating Processes, arXiv:1306. 3775 [INSPIRE].

[21] G. D'Ambrosio, G.F. Giudice, G. Isidori and A. Strumia, Minimal flavor violation: An Effective field theory approach, Nucl. Phys. B 645 (2002) 155 [hep-ph/0207036] [INSPIRE].

[22] V. Cirigliano, B. Grinstein, G. Isidori and M.B. Wise, Minimal flavor violation in the lepton sector, Nucl. Phys. B 728 (2005) 121 [hep-ph/0507001] [INSPIRE].

[23] X.-G. He, C.-J. Lee, S.-F. Li and J. Tandean, Large electron electric dipole moment in minimal flavor violation framework with Majorana neutrinos, Phys. Rev. D 89 (2014) 091901 [arXiv:1401.2615] [InSPIRE].

[24] P. Minkowski, $\mu \rightarrow$ ey at a Rate of One Out of 1-Billion Muon Decays?, Phys. Lett. B 67 (1977) 421 [INSPIRE].

[25] T. Yanagida, Horizontal gauge symmetry and masses of neutrinos, in Proceedings of the Workshop on the Unified Theory and the Baryon Number in the Universe, O. Sawada and A. Sugamoto eds., KEK, Tsukuba, 1979, pg. 95.

[26] T. Yanagida, Horizontal Symmetry and Masses of Neutrinos, Prog. Theor. Phys. 64 (1980) 1103 [InSPIRE].

[27] M. Gell-Mann, P. Ramond and R. Slansky, Complex spinors and unified theories, in Supergravity, P. van Nieuwenhuizen and D. Freedman eds., North-Holland, Amsterdam, 1979, pg. 315 .

[28] P. Ramond, The Family Group in Grand Unified Theories, hep-ph/9809459 [InSPIRE].

[29] S.L. Glashow, The future of elementary particle physics, in Proceedings of the 1979 Cargese Summer Institute on Quarks and Leptons, M. Levy and et al. eds., Plenum Press, New York, 1980, pg. 687.

[30] R.N. Mohapatra and G. Senjanović, Neutrino Mass and Spontaneous Parity Violation, Phys. Rev. Lett. 44 (1980) 912 [InSPIRE].

[31] J. Schechter and J.W.F. Valle, Neutrino Masses in $\mathrm{SU}(2) \times U(1)$ Theories, Phys. Rev. D 22 (1980) 2227 [INSPIRE].

[32] J. Schechter and J.W.F. Valle, Neutrino Decay and Spontaneous Violation of Lepton Number, Phys. Rev. D 25 (1982) 774 [inSPIRE]. 
[33] R. Foot, H. Lew, X.G. He and G.C. Joshi, Seesaw Neutrino Masses Induced by a Triplet of Leptons, Z. Phys. C 44 (1989) 441 [inSPIRE].

[34] G. Colangelo, E. Nikolidakis and C. Smith, Supersymmetric models with minimal flavour violation and their running, Eur. Phys. J. C 59 (2009) 75 [arXiv:0807.0801] [InSPIRE].

[35] L. Mercolli and C. Smith, EDM constraints on flavored CP-violating phases, Nucl. Phys. B 817 (2009) 1 [arXiv:0902.1949] [InSPIRE].

[36] J.A. Casas and A. Ibarra, Oscillating neutrinos and $\mu \rightarrow e, \gamma$, Nucl. Phys. B 618 (2001) 171 [hep-ph/0103065] [INSPIRE].

[37] V. Cirigliano, G. Isidori and V. Porretti, CP violation and Leptogenesis in models with Minimal Lepton Flavour Violation, Nucl. Phys. B 763 (2007) 228 [hep-ph/0607068] [INSPIRE].

[38] Z.-z. Xing, H. Zhang and S. Zhou, Impacts of the Higgs mass on vacuum stability, running fermion masses and two-body Higgs decays, Phys. Rev. D 86 (2012) 013013 [arXiv:1112.3112] [INSPIRE].

[39] CKMfitTeR group, http://ckmfitter.in2p3.fr.

[40] G. Degrassi, E. Franco, S. Marchetti and L. Silvestrini, QCD corrections to the electric dipole moment of the neutron in the MSSM, JHEP 11 (2005) 044 [hep-ph/0510137] [INSPIRE].

[41] C. Dib, A. Faessler, T. Gutsche, S. Kovalenko, J. Kuckei et al., The Neutron electric dipole form-factor in the perturbative chiral quark model, J. Phys. G 32 (2006) 547 [hep-ph/0601144] [INSPIRE].

[42] G. 't Hooft, Symmetry Breaking Through Bell-Jackiw Anomalies, Phys. Rev. Lett. 37 (1976) 8 [inSPIRE].

[43] R. Jackiw and C. Rebbi, Vacuum Periodicity in a Yang-Mills Quantum Theory, Phys. Rev. Lett. 37 (1976) 172 [INSPIRE].

[44] C.G. Callan Jr., R.F. Dashen and D.J. Gross, The Structure of the Gauge Theory Vacuum, Phys. Lett. B 63 (1976) 334 [InSPIRE].

[45] F. Capozzi, G.L. Fogli, E. Lisi, A. Marrone, D. Montanino et al., Status of three-neutrino oscillation parameters, circa 2013, Phys. Rev. D 89 (2014) 093018 [arXiv:1312.2878] [INSPIRE].

[46] W. Rodejohann, Neutrinoless double beta decay and neutrino physics, J. Phys. G 39 (2012) 124008 [arXiv:1206.2560] [INSPIRE].

[47] S.T. Petcov, Leptonic CP-violation and leptogenesis, Int. J. Mod. Phys. A 29 (2014) 1430028 [arXiv:1405.2263] [InSPIRE].

[48] Planck collaboration, P.A.R. Ade et al., Planck 2013 results. XVI. Cosmological parameters, arXiv:1303.5076 [INSPIRE].

[49] S. Riemer-Sørensen, D. Parkinson and T.M. Davis, Combining Planck with Large Scale Structure gives strong neutrino mass constraint, arXiv:1306.4153 [INSPIRE].

[50] Z. Hou, C.L. Reichardt, K.T. Story, B. Follin, R. Keisler et al., Constraints on Cosmology from the Cosmic Microwave Background Power Spectrum of the 2500-square degree SPT-SZ Survey, Astrophys. J. 782 (2014) 74 [arXiv:1212.6267] [INSPIRE]. 
[51] BOSS collaboration, F. Beutler et al., The clustering of galaxies in the SDSS-III Baryon Oscillation Spectroscopic Survey: Signs of neutrino mass in current cosmological datasets,

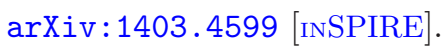

[52] S. Kanemura, T. Kasai and Y. Okada, Mass bounds of the lightest CP even Higgs boson in the two Higgs doublet model, Phys. Lett. B 471 (1999) 182 [hep-ph/9903289] [InSPIRE].

[53] KamLAND-Zen collaboration, A. Gando et al., Limit on Neutrinoless $\beta \beta$ Decay of Xe-136 from the First Phase of KamLAND-Zen and Comparison with the Positive Claim in Ge-76, Phys. Rev. Lett. 110 (2013) 062502 [arXiv:1211.3863] [INSPIRE].

[54] GERDA collaboration, M. Agostini et al., Results on Neutrinoless Double- $\beta$ Decay of ${ }^{76}$ Ge from Phase I of the GERDA Experiment, Phys. Rev. Lett. 111 (2013) 122503 [arXiv: 1307.4720] [INSPIRE].

[55] NEMO-3 collaboration, R. Arnold et al., Search for Neutrinoless Double-Beta Decay of ${ }^{100}$ Mo with the NEMO-3 Detector, Phys. Rev. D 89 (2014) 111101 [arXiv:1311.5695] [INSPIRE].

[56] EXO-200 collaboration, J.B. Albert et al., Search for Majorana neutrinos with the first two years of EXO-200 data, Nature 510 (2014) 229 [arXiv:1402.6956] [INSPIRE].

[57] M. Vignati, Model of the Response Function of CUORE Bolometers, EPJ Web Conf. 70 (2014) 00044.

[58] Z.-z. Xing, Implications of generalized Frampton-Glashow-Yanagida ansaetze on neutrino masses and lepton flavor mixing, Phys. Rev. D 69 (2004) 013006 [hep-ph/0307007] [INSPIRE].

[59] X.-G. He and W. Liao, A Light Sterile Neutrino from Friedberg-Lee Symmetry, Phys. Lett. B 728 (2014) 68 [arXiv:1309.7581] [INSPIRE].

[60] Muon (G-2) collaboration, G.W. Bennett et al., An Improved Limit on the Muon Electric Dipole Moment, Phys. Rev. D 80 (2009) 052008 [arXiv:0811.1207] [InSPIRE].

[61] W.C. Griffith, M.D. Swallows, T.H. Loftus, M.V. Romalis, B.R. Heckel et al., Improved Limit on the Permanent Electric Dipole Moment of Hg-199, Phys. Rev. Lett. 102 (2009) 101601 [INSPIRE].

[62] X.-G. He, T. Li, X.-Q. Li, J. Tandean and H.-C. Tsai, Constraints on Scalar Dark Matter from Direct Experimental Searches, Phys. Rev. D 79 (2009) 023521 [arXiv:0811.0658] [INSPIRE].

[63] X.-G. He, B. Ren and J. Tandean, Hints of Standard Model Higgs Boson at the LHC and Light Dark Matter Searches, Phys. Rev. D 85 (2012) 093019 [arXiv:1112.6364] [INSPIRE].

[64] R.D. Young and A.W. Thomas, Recent results on nucleon sigma terms in lattice QCD, Nucl. Phys. A 844 (2010) 266C [arXiv:0911.1757] [InSPIRE].

[65] P. Junnarkar and A. Walker-Loud, Scalar strange content of the nucleon from lattice QCD, Phys. Rev. D 87 (2013) 114510 [arXiv:1301.1114] [INSPIRE].

[66] J.M. Alarcon, L.S. Geng, J. Martin Camalich and J.A. Oller, The strangeness content of the nucleon from effective field theory and phenomenology, Phys. Lett. B 730 (2014) 342 [arXiv: 1209.2870] [INSPIRE].

[67] R. Ruiz de Austri and C. Pérez de los Heros, Impact of nucleon matrix element uncertainties 
on the interpretation of direct and indirect dark matter search results, JCAP 11 (2013) 049 [arXiv: 1307.6668] [INSPIRE].

[68] C.A. Aidala, S.D. Bass, D. Hasch and G.K. Mallot, The Spin Structure of the Nucleon, Rev. Mod. Phys. 85 (2013) 655 [arXiv:1209.2803] [InSPIRE].

[69] T. Aoyama, M. Hayakawa, T. Kinoshita and M. Nio, Complete Tenth-Order QED Contribution to the Muon g-2, Phys. Rev. Lett. 109 (2012) 111808 [arXiv:1205.5370] [INSPIRE].

[70] MEG collaboration, J. Adam et al., New constraint on the existence of the $\mu^{+} \rightarrow e^{+} \gamma$ decay, Phys. Rev. Lett. 110 (2013) 201801 [arXiv: 1303.0754] [INSPIRE].

[71] A. de Gouvêa and P. Vogel, Lepton Flavor and Number Conservation and Physics Beyond the Standard Model, Prog. Part. Nucl. Phys. 71 (2013) 75 [arXiv:1303.4097] [InSPIRE].

[72] R. Kitano, M. Koike and Y. Okada, Detailed calculation of lepton flavor violating muon electron conversion rate for various nuclei, Phys. Rev. D 66 (2002) 096002 [Erratum ibid. D 76 (2007) 059902] [hep-ph/0203110] [INSPIRE].

[73] D.K. Papoulias and T.S. Kosmas, Nuclear aspects of neutral current non-standard $\nu$-nucleus reactions and the role of the exotic $\mu^{-} \rightarrow e^{-}$transitions experimental limits, Phys. Lett. B 728 (2014) 482 [arXiv:1312.2460] [INSPIRE].

[74] Heavy Flavor Averaging Group collaboration, Y. Amhis et al., Averages of B-Hadron, C-Hadron and tau-lepton properties as of early 2012, arXiv:1207.1158 [INSPIRE].

[75] http://www.slac.stanford.edu/xorg/hfag.

[76] M. Misiak and M. Steinhauser, Nucl. Phys. B 764 (2007) 62 [hep-ph/0609241]. 